\title{
Evaluating the role of electricity storage by considering short-term operation in long-term planning
}

\author{
Tom Brijs ${ }^{\mathrm{a}, \mathrm{b}, *}$, Arne van Stiphout ${ }^{\mathrm{a}}$, Sauleh Siddiqui ${ }^{\mathrm{b}, \mathrm{c}, \mathrm{d}}$, Ronnie Belmans ${ }^{\mathrm{a}}$ \\ ${ }^{a}$ Department of Electrical Engineering, University of Leuven (KU Leuven), Heverlee, Belgium, and \\ EnergyVille Research Institute, Genk, Belgium \\ ${ }^{b}$ Department of Civil Engineering, The Johns Hopkins University, Baltimore, MD, USA \\ ${ }^{c}$ Department of Applied Mathematics and Statistics, The Johns Hopkins University, Baltimore, MD, \\ $U S A$ \\ ${ }^{d}$ German Institute for Economic Research (DIW Berlin), Berlin, Germany
}

\begin{abstract}
Short-term operating requirements and constraints in power systems are becoming increasingly important with the greater flexibility needed due to the integration of variable renewables. However, large problem sizes and computational barriers have limited the extent to which they are included in long-term planning models. Our objective is to understand the role of electricity storage in future renewable-based systems by including an accurate representation of short-term operation with high temporal detail within a long-term planning framework. Specifically, we discuss the development of a long-term investment model including a continuous relaxation of the technology-clustered formulation of the short-term unit commitment problem, including detailed operating reserve sizing and supply. This model is solved for a full year, and is applied to a test system with system load and renewable generation characteristics from the Belgian power system in a greenfield setting, i.e., assuming no pre-existing capacities, to analyze the role of storage at different renewable penetration levels. Both pumped-hydro storage and battery energy storage is considered, and their role in providing energy services and frequency control is investigated. We derive broadly applicable conclusions on the benefits and role of electricity storage to motivate why it may be built and operated. Results show that, in general, the integration of storage resources decreases total system cost, partially replaces flexible power plants, facilitates the integration of renewable energy sources, and allows inflexible technologies to perform better.
\end{abstract}

Keywords: Electricity storage, Renewable energy, Power system flexibility, Long-term power system planning, Short-term power system operation

\footnotetext{
* Corresponding author

Email addresses: tom.brijs@esat.kuleuven.be (Tom Brijs), arne.vanstiphout@esat.kuleuven.be (Arne van Stiphout), siddiqui@jhu.edu (Sauleh Siddiqui), ronnie.belmans@esat.kuleuven.be (Ronnie Belmans)
} 


\section{Introduction}

\subsection{Motivation}

Electricity storage refers to systems, bidirectionally coupled with the power system, which buffer energy. The energy buffer can be implemented based on a variety of physi-

5 cal principles: energy stored as thermal, chemical, electro-chemical, kinetic, or potential energy, or in the electro-magnetic field [1]. Although pumped-hydro storage (PHS) is currently the most installed storage technology with $\geq 99 \%$ of global capacity, and significant potential for new PHS capacity may still be present $[2,3]$, cost decreases and technological advancements are making battery energy storage (BES) increasingly com-

10 petitive. The storage of electricity is expected to play an important role in the transition to power systems with high shares of variable renewable energy sources (RES) in the generation mix [4]. Variable RES technologies, i.e., wind turbines and photovoltaic (PV) systems, are characterized by their dependency on weather conditions, which lead to expected power variations and unexpected forecast errors, and as such generally increase

15 the variability in the system. This variability can be dealt with by flexibility, which indicates the ability to provide power adjustments to keep the system balanced at different time scales. Flexibility can be provided by flexible supply, flexible demand, and storage, which can also be activated in neighboring regions through the grid [5].

This article studies the role of storage in future RES-based systems by means of a long-term investment model for generation and storage capacity, commonly referred to as a generation expansion planning (GEP) model. Conventional GEP models typically include a simplified representation of short-term system operation and its costs, through low temporal and operational detail. However, the ongoing integration of variable RES makes short-term system operation increasingly important to consider in detail in longterm system planning [6,7]. It is only by considering a high level of temporal and operational detail that accurate insights can be obtained in the role of storage.

\subsection{Literature review}

\subsubsection{Including temporal and operational detail in generation expansion planning}

Four approaches can be identified for the temporal detail, each with a different trade30 off between computation time and the extent to which short-term operation can be accurately considered. First, planners can use a load duration curve (LDC), or residual LDC (RLDC) when considering variable RES. This approach neglects the chronology of the generation and consumption balance, and thus not allows to include intertemporal links and related costs (i.e., start-up and shut-down costs, minimum up and down times,

35 ramp rates and costs, and energy storage buffer dynamics) [8]. Second, some GEP models represent the seasonal, weekly, and daily variability within a year by a limited number of time slices [9]. The value for any time slice for the RES generation and system load corresponds to the average value in the period considered in the time slice. While this may not result in large approximation errors with low shares of variable RES, with high shares

40 of variable RES this underestimates the variability of the residual load, and undervalues flexible technologies compared to inflexible technologies [10, 11]. Third, GEP models can include a set of representative periods (e.g., a few days or weeks), chosen such that their RLDC approaches the RLDC of the entire year $[8,10]$. The disadvantage is that the selection of representative periods may be difficult to justify, and that it is difficult 
45 to represent all variability in the system in a limited set of periods, thereby leading to inaccuracies [9]. Fourth, the most accurate approach, but also the most computationally intensive one, is the use of hourly time steps for the entire year. While this is common practice in short-term models, it is not for long-term models due to the increase in problem size.

The operational detail determines how accurately technical constraints on the power plant level (i.e., commitment decisions, generation limits, ramp rates, minimum up and down times, energy storage buffer dynamics) and system level (i.e., power balance, reserve balance) are taken into account. High operational detail is typically present in shortterm power system models, i.e., unit commitment (UC) and economic dispatch models,

55 which can assess the impact of RES integration on system operation, and thus on the scheduling of power plants. They are generally used to focus on issues related to flexibility adequacy, i.e., the short-term ability to keep the system balanced. In contrast, longterm power system models, i.e., investment and GEP models, can assess the impact of RES integration on system planning, and thus on the generation mix. They are so generally used to focus on issues related to system adequacy, i.e., the ability to meet peak demand. To keep computation efforts within limits, they typically do not consider the same level of operational detail as short-term models [12]. Historically, low operational detail could be present in GEP models without large approximation errors [13, 14]. However, this is not the case with the greater flexibility required due to the integration 65 of variable RES: short-term operation becomes increasingly important to consider in long-term planning [15]. Not considering this leads to an underestimation of the need for and value of flexibility $[6,7]$. In this regard, two approaches to increase operational detail in GEP can be identified [10]. In the first approach, the investment and operation problem are considered separately and sequentially, i.e., the results of the investment 70 problem are ex post used as input for the operation problem. This is referred to as the so-called soft-linking of both problems, which can also be solved iteratively [16]. In the second approach, the operation problem with high operational detail and the investment problem are solved together and simultaneously.

\subsubsection{The role of electricity storage in power systems}

75

The role and value of storage has already been studied in the existing literature with varying levels of capacity expansion opportunities and temporal and operational detail.

First, many studies have examined the benefits of storage on system operation by considering predetermined generation and storage portfolios, not allowing for endogenous capacity expansions (e.g., [17, 18, 19, 20, 21, 22]). This is typically done by comso paring results, in terms of operational costs, dispatch schedules, or RES curtailment, of a reference case without storage with one or multiple cases including storage.

Second, others focus exclusively on the storage sizing problem given exogenouslydefined generation portfolios. Ref. [23] optimizes storage investments considering the residual load and using a simplified representation of short-term operation, with storage 35 as a way to compensate excess RES generation. In [24] storage sizing is based on the system's variability, while in [25] it is based on the system's uncertainty, and [26, 27, 28] consider both to determine storage requirements over different time scales.

Third, the final category of studies co-optimizes storage with generation investments. Ref. [29] uses some form of RLDC method, neglecting the chronology of the required power balance over time, thereby not capturing intertemporal links and related costs. 
Ref. [30] does not consider any operational detail in the planning of storage, generation, and grid capacity. Ref. [31] considers an hourly power balance, subject to operational constraints, but does not consider reserve requirements. In [32] a detailed combined investment and dispatch model is proposed, which neglects commitment decisions, min-

95 imum load levels, and minimum up and down times, but aims to compensate this shortcoming through ramping penalties. Ref. [33] includes a detailed representation of system operation, but considers exogenous investments in RES and endogenous investments in selected conventional generation technologies and compressed air energy storage, and includes simplified reserve modeling. In [34] and [35, 36] a detailed short-term operation is included, but only a limited number of representative days is considered. This may lead to inaccurate representations of consumption and RES generation variations, and may not fully capture the added value of the ability of mid-to-long-term storage to shift energy between more distant, or longer, periods of time. Finally, [37] includes a lot of operational detail, but only considers four representative weeks, and assumes fixed energy-to-power (E2P) ratios for storage, making it difficult to gain insight in optimal storage sizing for energy-related vs. power-related services. In addition, whereas we focus on RES generation targets, [37] focuses on $\mathrm{CO}_{2}$ emission goals.

\subsection{Scope and contributions}

This article's scope and contribution is the illustration of the role of electricity storage in future RES-based systems by including an accurate representation of short-term system operation within a long-term planning framework. We first discuss the development of a combined, and simultaneously solved, long-term investment and short-term operation model with high temporal and operational detail. We consider hourly periods for a full year, and consider high operational detail in line with what is considered in a 115 short-term power system model. In order to be able to solve numerically for meaningful optimization horizons, short-term operation is modeled through a continuously-relaxed and technology-clustered approximation of the conventional mixed-integer plant-level UC problem. This model is able to capture the increasing impact of flexibility needs in both the close-to-real-time scheduling phase (i.e., energy market) and real-time operation phase (i.e., reserve market), following the ongoing integration of variable RES, and includes a detailed representation of the flexibility supply by both generation and storage technologies. The representation of operating reserves is unparalled in that it includes detailed exogenous and endogenous reserve sizing, and periodical (i.e., for multiple hourly periods) reserve allocation to providers, in line with the market design in

125 Europe. Second, we apply this model to a test system with system load and RES generation characteristics from the Belgian power system in a greenfield setting, i.e., assuming no pre-existing capacities. We do not aim to determine likely deployment scenarios or address optimal pathways towards the future, but to derive general conclusions on the benefits and role of storage at different RES penetration levels, and to gain insight in

130 the interdependency between flexibility options. Both PHS and BES is considered, and their role in providing energy services and frequency control is investigated. We consider different storage scenarios with regard to the available natural potential for PHS and decreasing cost of the energy storage subsystem of BES. 


\section{Methodology}

The developed model is a partial equilibrium model, focusing solely on the electricity sector. It decides on the investments in and dispatch of generation and storage capacity to meet the demand for energy and reserves at lowest total system cost, while respecting detailed short-term operation constraints, and reaching increasing RES targets. Results from such a system perspective approach may serve as a proxy for the outcome in a vertically integrated environment, an unbundled environment with a centralized electricity pool model (e.g., the PJM market), or a liberalized market with bilateral- and exchange-based trading (e.g., the European market) assuming perfect competition [38].

All technologies are defined as either injection or offtake technologies. In the developed model injection technologies includes dispatchable and intermittent generation, and storage discharging. In real power systems this also includes import from adjacent markets through interconnection capacity, and the postponing of flexible consumption. Offtake technologies include storage charging, but in real systems this also includes export to adjacent markets and the forwarding of flexible consumption. Table 1, Table 2, and Table 3 provide an overview of the sets, decision variables, and input parameters used in this article, and their SI (or base) and most commonly used unit.

\subsection{Continuously-relaxed and technology-clustered UC formulation}

The mixed-integer plant-level UC problem alone is already computationally challenging to solve for significant optimization horizons, even in its deterministic form and without grid representation. This is in part due to its many and elaborate techno-economic constraints, but especially because of the many commitment decisions represented by binary variables for each unit for each time step. Therefore, a more computational-friendly formulation of the UC problem needs to be included as a subproblem next to the investment problem within a GEP model with high temporal detail. To reduce problem size and facilitate manageable computation times, we couple a continuous relaxation of the technology-clustered formulation of the UC problem to the investment problem. ${ }^{1}$

\subsubsection{Technology-clustered UC formulation}

A technology-clustered formulation combines identical or similar units into clusters, which assumes non-binding transmission constraints, i.e., a copper plate, and identical techno-economic characteristics of units within a cluster. The latter introduces approximation errors for existing capacity, as for this capacity differences among units exist due to project-specific elements, but not for new capacity, as for this capacity it is common practice in planning studies to use generalized data. The use of technology-generalized data thus impacts the results in long-term GEP with capacity legacy, but does not introduce errors compared to plant-level formulations in long-term greenfield GEP [8, 12, 39]. Approximation errors occur because of the inherent mathematical difference between plant-level and clustered formulations. However, $[8,39]$ show that a clustered formulation only results in minimal errors (i.e., near or below 1\%) while needing significantly less computation time (i.e., up to 2000 times), which is also confirmed by [40], thereby justifying the use of a technology-clustered UC formulation in GEP models.

\footnotetext{
${ }^{1}$ Although this continuously-relaxed and technology-clustered approximation should not be used to analyze actual system operation, it is valuable to include short-term operation in long-term planning.
} 
Clustering reduces the problem size in two ways. First, the large set of binary variables representing the commitment decision of individual units (i.e., 0 or 1 ) is replaced by a smaller set of integer variables that represent the commitment decision of a cluster (i.e., from 0 to the number of units in the cluster). Second, clustering also reduces the number of continuous equations and variables, as all decisions except a unit's commitment (i.e.,

180 power output, reserve provision) apply to the small number of clusters rather than the large set of individual units. Commitment decisions are still captured at the unit level.

\subsubsection{Continuously-relaxed UC formulation}

In addition, to include the UC problem in an investment framework, computation times are further reduced by replacing the integer commitment variables by linear commitment variables. This linearized technology-clustered formulation of the short-term UC problem coupled to the long-term investment problem has already been succesfully used in $[6,41]$. Of all short-term operating constraints, [41] found that relaxing integers provides the best accuracy vs. computation time trade-offs for power system planning purposes, and concludes that a continuously-relaxed and technology-clustered formulation is strongly advised for GEP studies focusing on flexibility.

Table 1: Nomenclature: list of sets.

\begin{tabular}{|c|c|}
\hline Symbol & Description \\
\hline$h \in \mathbb{H}$ & Hourly time steps. \\
\hline$i \in \mathbb{I}$ & Injection technologies, $\mathbb{I}=\mathbb{I} \mathbb{D} \cup \mathbb{I I}$. \\
\hline $\mathbb{I D}$ & Dispatchable injection technologies, $\mathbb{I D} \subseteq \mathbb{I}$. \\
\hline $\mathbb{I I I}$ & Intermittent injection technologies, $\mathbb{I I} \subseteq \mathbb{I}$. \\
\hline$o \in \mathbb{O}$ & Offtake technologies, $\mathbb{O}=\mathbb{S}$. \\
\hline$r \in \mathbb{R}$ & Operating reserve categories, $\mathbb{R}=\mathbb{R U} \cup \mathbb{R} \mathbb{D}$. \\
\hline $\mathbb{R D}$ & Downward FCR, aFRR, and $\mathrm{mFRR}, \mathbb{R D} \subset \mathbb{R}$. \\
\hline $\mathbb{R D A}$ & Downward FCR and aFRR, $\mathbb{R D A} \subset \mathbb{R D}$. \\
\hline $\mathbb{R D F}$ & Downward FCR, $\mathbb{R D F} \subset \mathbb{R D A}$. \\
\hline $\mathbb{R U}$ & Upward FCR, aFRR, and mFRR, $\mathbb{R} \mathbb{U} \subset \mathbb{R}$. \\
\hline $\mathbb{R U A}$ & Upward FCR and aFRR, $\mathbb{R U A} \subset \mathbb{R} U$. \\
\hline $\mathbb{R} \mathbb{E}$ & Upward FCR, $\mathbb{R U F} \subset \mathbb{R U A}$ \\
\hline$s \in \mathbb{S}$ & Electricity storage technologies, $\mathbb{S} \subseteq \mathbb{I D}, \mathbb{S}=\mathbb{O}$. \\
\hline$w \in \mathbb{W}$ & Minimum up time set. \\
\hline$z \in \mathbb{Z}$ & Minimum down time set. \\
\hline
\end{tabular}

\subsection{Objective function}

The objective of the developed model is to determine the generation and storage mix, output schedules, and reserve provision, such that the demand for energy and reserve capacity is met at the lowest total system cost over the full optimization horizon ${ }_{195}|\mathbb{H}| \cdot T^{\mathrm{h}}(1)$. The total system cost consists of the following costs for all injection and offtake technologies: the power-related investment cost, the fixed operating and maintenance $(\mathrm{O} \& \mathrm{M})$ cost, the fuel cost, the variable O\&M cost, the ramp cost, the startup cost, and the shut-down cost; the following additional costs for all storage technologies: the energy-related investment cost, and the depreciation cost following excessive storage cycling; and finally the load shedding cost in case of insufficient available supply, and the curtailment cost in case of excess renewable generation: 


$$
\begin{aligned}
& \min \left[\sum _ { i \in \mathbb { I } } \left[\left(C_{i}^{\mathrm{inv}, \text { inj }}+C_{i}^{\mathrm{fom}, \mathrm{inj}}\right) \cdot p_{i}^{\mathrm{inst}, \mathrm{inj}}+\sum_{h \in \mathbb{H}}\left[\left(C_{i}^{\mathrm{fuel}, \mathrm{inj}}+C_{i}^{\mathrm{vom}, \mathrm{inj}}\right) \cdot p_{i, h}^{\mathrm{inj}} \cdot T^{\mathrm{h}}\right.\right.\right. \\
& \left.\left.+C_{i}^{\mathrm{ra}, \mathrm{inj}} \cdot\left(p_{i, h}^{\mathrm{ru}, \mathrm{inj}}+p_{i, h}^{\mathrm{rd}, \mathrm{inj}}\right)+C_{i}^{\mathrm{su}, \mathrm{inj}} \cdot p_{i, h}^{\mathrm{su}, \mathrm{inj}}+C_{i}^{\mathrm{sd}, \mathrm{inj}} \cdot p_{i, h}^{\mathrm{sd}, \mathrm{inj} j}\right]\right] \\
& +\sum_{o \in \mathbb{O}}\left[\left(C_{o}^{\mathrm{inv}, \mathrm{off}}+C_{o}^{\mathrm{fom}, \mathrm{off}}\right) \cdot p_{o}^{\mathrm{inst}, \mathrm{off}}+\sum_{h \in \mathbb{H}}\left[\left(C_{o}^{\mathrm{fuel}, \mathrm{off}}+C_{o}^{\mathrm{vom}, \mathrm{off}}\right) \cdot p_{o, h}^{\mathrm{off}} \cdot T^{\mathrm{h}}\right.\right. \\
& \left.\left.\left.+C_{o}^{\mathrm{ra}, \mathrm{off}} \cdot\left(p_{o, h}^{\mathrm{ru}, \mathrm{off}}+p_{o, h}^{\mathrm{rd}, \mathrm{off}}\right)+C_{o}^{\mathrm{su}, \mathrm{off}} \cdot p_{o, h}^{\mathrm{su}, \mathrm{off}}+C_{o}^{\mathrm{sd}, \mathrm{off}} \cdot p_{o, h}^{\mathrm{sd}, \mathrm{off}}\right)\right]\right] \\
& \left.+\sum_{s \in \mathbb{S}}\left(C_{s}^{\mathrm{inv}, \mathrm{e}} \cdot e_{s}^{\mathrm{inst}}\right)+\sum_{h \in \mathbb{H}}\left(C^{\mathrm{ls}} \cdot p_{h}^{\mathrm{ls}}+\sum_{i \in \mathbb{I}} C_{i}^{\mathrm{cu}} \cdot p_{i, h}^{\mathrm{cu}, \mathrm{inj}}\right)\right] /\left(|\mathbb{H}| \cdot T^{\mathrm{h}}\right)+\sum_{s \in \mathbb{S}} c_{s}^{\mathrm{cyc}}
\end{aligned}
$$

\begin{tabular}{|c|c|c|}
\hline Symbol & Unit (SI, typical) & Description \\
\hline$c_{s}^{\text {cyc }}$ & $€ / \mathrm{s}, € /$ year & Cycling cost of technology $s$. \\
\hline$e_{s, h}$ & J, MWh & Stored energy level of technology $s$ at time step $h$. \\
\hline$e_{s}^{\text {inst }}$ & $\mathrm{J}, \mathrm{MWh}$ & Installed energy storage capacity of technology $s$. \\
\hline$n_{i, h}^{\text {inj }}, n_{o, h}^{\text {off }}$ & - & Number of online units of technology $i$ and $o$ at time step $h$. \\
\hline$n_{i, h}^{\text {sd,inj }}, n_{o, h}^{\text {sd, off }}$ & - & Number of online units of technology $i$ and $o$ shutting down at time step $i$ \\
\hline$n_{r, i, h}^{\text {sdr,inj }}, n_{r, o, h}^{\text {sdr,off }}$ & - & $\begin{array}{l}\text { Number of online units of technology } i \text { and } o \text { committed to shut down } \\
\text { at time step } h \text { to provide reserve } r \text {. }\end{array}$ \\
\hline$n_{i, h}^{\mathrm{su}, \mathrm{inj}}, n_{o, h}^{\mathrm{su} \text { off }}$ & - & Number of offline units of technology $i$ and $o$ starting up at time step $h$. \\
\hline$n_{r, i, h}^{\text {sur,inj }}, n_{r, o, h}^{\text {sur,off }}$ & - & $\begin{array}{l}\text { Number of offline units of technology } i \text { and } o \text { committed to start up at } \\
\text { time step } h \text { to provide reserve } r \text {. }\end{array}$ \\
\hline$p_{i, h}^{\mathrm{cu}, \mathrm{inj}}$ & $\mathrm{W}, \mathrm{MW}$ & Curtailment of technology $i$ at time step $h$. \\
\hline$p_{i, h}^{\text {inj }}, p_{o, h}^{\text {off }}$ & $\mathrm{W}, \mathrm{MW}$ & Injection of technology $i$ and offtake of technology $o$ at time step $h$. \\
\hline$p_{i}^{\text {inst, inj }}, p_{o}^{\text {inst,off }}$ & W, MW & Installed power rating of technology $i$ and $o$. \\
\hline$p_{h}^{\mathrm{ls}}$ & $\mathrm{W}, \mathrm{MW}$ & Load shedding at time step $h$. \\
\hline$p_{i, h}^{\mathrm{rd}, \mathrm{inj}}, p_{o, h}^{\mathrm{rd}, \mathrm{off}}$ & $\mathrm{W}, \mathrm{MW}$ & $\begin{array}{l}\text { Decrease in injection of technology } i \text { and offtake of technology } o \text { by } \\
\text { ramping down units at time step } h \text {. }\end{array}$ \\
\hline$p_{i, h}^{\mathrm{ru}, \mathrm{inj}}, p_{o, h}^{\mathrm{ru}, \text { off }}$ & $\mathrm{W}, \mathrm{MW}$ & $\begin{array}{l}\text { Increase in injection of technology } i \text { and offtake of technology } o \text { by } \\
\text { ramping up units at time step } h \text {. }\end{array}$ \\
\hline$p_{i, h}^{\text {sd,inj }}, p_{o, h}^{\text {sd,off }}$ & W, MW & $\begin{array}{l}\text { Decrease in injection of technology } i \text { and offtake of technology } o \text { by } \\
\text { shutting down units at time step } h \text {. }\end{array}$ \\
\hline$p_{i, h}^{\mathrm{su}, \mathrm{inj}}, p_{o, h}^{\mathrm{su}, \mathrm{off}}$ & $\mathrm{W}, \mathrm{MW}$ & $\begin{array}{l}\text { Increase in injection of technology } i \text { and offtake of technology } o \text { by } \\
\text { starting up units at time step } h .\end{array}$ \\
\hline$r_{r, i, h}^{\mathrm{inj}}, r_{r, o, h}^{\mathrm{off}}$ & $\mathrm{W}, \mathrm{MW}$ & Provision of reserve $r$ by technology $i$ and $o$ at time step $h$. \\
\hline$r_{r, i, h}^{\mathrm{s}, \text { inj }}, r_{r, o, h}^{\mathrm{s} \text { off }}$ & W, MW & Provision of reserve $r$ by online units of technology $i$ and $o$ at time step $h$. \\
\hline$r_{r, i, h}^{\mathrm{sd}, i n j}, r_{r, o, h}^{\mathrm{sd}, \text { off }}$ & $\mathrm{W}, \mathrm{MW}$ & $\begin{array}{l}\text { Provision of reserve } r \text { by online units of technology } i \text { and } o \\
\text { by shutting down at time step } h \text {. }\end{array}$ \\
\hline$r_{r, i, h}^{\mathrm{su}, \mathrm{inj}}, r_{r, o, h}^{\mathrm{su}, \mathrm{off}}$ & $\mathrm{W}, \mathrm{MW}$ & $\begin{array}{l}\text { Provision of reserve } r \text { by offline units of technology } i \text { and } o \\
\text { by starting up at time step } h .\end{array}$ \\
\hline
\end{tabular}

Table 2: Nomenclature: list of decision variables (all are nonnegative, i.e., $\in \mathbb{R}_{+}$).

\subsection{Power system constraints}

Three requirements are considered on the system level. First, an hourly power balance between scheduled generation and consumption is included, i.e., the energy marketclearing constraint (2a). This ensures that the expected variability in the system is dealt with. Second, an hourly balance between the demand for and supply of reserve capacity is included, i.e., the reserve market-clearing constraint (2b). This ensures that the 
Table 3: Nomenclature: list of parameters.

\begin{tabular}{|c|c|c|}
\hline Symbol & Unit (SI, typical) & Description \\
\hline$A_{i, h}^{\text {res }}$ & $\%$ & Generation forecast for intermittent injection technology $i$. \\
\hline$C_{i}^{c, n}$ & $€ / \mathrm{W}, € / \mathrm{MW}$ & Curtailment cost of technology $i$. \\
\hline$C_{i}^{\mathrm{fom}, \mathrm{inj}}, C_{o}^{\mathrm{fom}, \mathrm{off}}$ & $€ / \mathrm{W}, € / \mathrm{MW}$ & Fixed O\&M cost of technology $i$ and $o$. \\
\hline$C_{i}^{\text {fuel,inj }}, C_{o}^{\text {fuel,off }}$ & $€ / J, € / M W h$ & Fuel cost of technology $i$ and $o$. \\
\hline$C_{s}^{\text {inv, } \mathrm{e}}$ & $€ / J, € / M W h$ & Investment cost for energy capacity of technology $s$. \\
\hline $\begin{array}{c}C_{i}^{\mathrm{inv}, \text { inj }}, C_{o}^{\mathrm{inv}, \mathrm{off}} \\
C^{\mathrm{ls}}\end{array}$ & $\begin{array}{l}€ / \mathrm{W}, € / \mathrm{MW} \\
€ / \mathrm{W}, € / \mathrm{MW}\end{array}$ & $\begin{array}{l}\text { Investment cost for power rating of technology } i \text { and } o \text {. } \\
\text { Load shedding cost. }\end{array}$ \\
\hline$C_{i}^{\mathrm{ra}, \text { inj }}, C_{o}^{\mathrm{ra}, \text { off }}$ & $€ / \mathrm{W}, € / \mathrm{MW}$ & Ramping cost of technology $i$ and $o$. \\
\hline$C_{i}^{\mathrm{sd}, \mathrm{inj}}, C_{o}^{\mathrm{sd}, \mathrm{off}}$ & $€ / \mathrm{W}, € / \mathrm{MW}$ & Shut-down cost of technology $i$ and $o$. \\
\hline$C_{i}^{\mathrm{su}, \text { inj }}, C_{o}^{\mathrm{su}, \mathrm{off}}$ & $€ / \mathrm{W}, € / \mathrm{MW}$ & Start-up cost of technology $i$ and $o$. \\
\hline $\begin{array}{c}C_{i}^{\mathrm{vom}, \text { inj }}, C_{o}^{\mathrm{vom}, \text { off }} \\
D_{h}\end{array}$ & $\begin{array}{l}€ / J, € / M W h \\
W, M W\end{array}$ & $\begin{array}{l}\text { Variable O\&M cost of technology } i \text { and } o . \\
\text { System load. }\end{array}$ \\
\hline $\begin{array}{l}N_{i}^{\mathrm{cal}, \text { inj }}, N_{o}^{\mathrm{cal}, \text { off }} \\
N^{\mathrm{cyc}}\end{array}$ & $\mathrm{s}$, years & $\begin{array}{l}\text { Calendar life of technology } i \text { and } o \text {. } \\
\text { Cycle-life of technology } s \text {. }\end{array}$ \\
\hline$P_{i}^{\text {inj }}, P^{\text {off }}$ & W, MW & Typical unit size of technology $i$ and $o$. \\
\hline $\begin{array}{l}P_{i}^{\min , \text { inj }}, P_{o}^{\min , \text { off }} \\
R_{r, i}^{\text {en }}\end{array}$ & $\begin{array}{l}\% \\
\%\end{array}$ & $\begin{array}{l}\text { Minimum load level of online units of technology } i \text { and } o \text {. } \\
\text { Endogenous reserve requirement for reserve } r \text {. }\end{array}$ \\
\hline$R_{r}^{\text {ex }}$ & $\mathrm{W}, \mathrm{MW}$ & Exogenous reserve requirement for reserve $r$. \\
\hline$R_{i}^{\mathrm{m}, \mathrm{inj}}, R_{o}^{\mathrm{m}, \mathrm{off}}$ & $\% / \mathrm{s}, \% / \min$ & Ramping ability of technology $i$ and $o$ per minute. \\
\hline$R_{i}^{\mathrm{s}, \mathrm{inj}}, R_{o}^{\mathrm{s}, \text { off }}$ & $\%$ & Spinning ramping ability of technology $i$ and $o$ per time step. \\
\hline$R_{r, i}^{\mathrm{s}, \mathrm{r}, \text { inj }}, R_{r, o}^{\mathrm{s}, \mathrm{r}, \mathrm{off}}$ & $\%$ & Ramping ability of technology $i$ and $o$ for reserve $r$. \\
\hline$R_{i}^{\mathrm{sd}, \mathrm{inj}}, R_{o}^{\mathrm{sd}, \mathrm{off}}$ & $\%$ & Shut-down ramping ability of technology $i$ and $o$ per time ste \\
\hline$R_{i}^{\mathrm{su}, \mathrm{inj}}, R_{o}^{\mathrm{su}, \mathrm{off}}$ & $\%$ & Start-up ramping ability of technology $i$ and $o$ per time step. \\
\hline$S^{\text {res }}$ & $\%$ & RES generation target. \\
\hline$T^{\mathrm{h}}$ & $\mathrm{s}, \mathrm{h}$ & Duration of time step $h$. \\
\hline$T_{i}^{\mathrm{mdt}, \mathrm{inj}}, T_{o}^{\mathrm{mdt}, \mathrm{off}}$ & $\mathrm{s}, \mathrm{h}$ & Minimum down time of technology $i$ and $o$. \\
\hline$T_{i}^{\text {mut,inj }}, T_{o}^{\text {mut,off }}$ & $\mathrm{s}, \mathrm{h}$ & Minimum up time of technology $i$ and $o$. \\
\hline$T_{r}^{1, \mathrm{r}}$ & $\mathrm{s}, \min$ & Allowed ramp duration to provide reserve $r$. \\
\hline$T_{r}^{2, \mathrm{r}}$ & $\mathrm{s}, \mathrm{h}$ & Duration of the provision of reserve $r$ at contracted power. \\
\hline$\eta_{s}^{\text {inj }}, \eta_{s}^{\text {off }}$ & $\%$ & (Dis)charge efficiency of technology $s$. \\
\hline
\end{tabular}

unexpected variability in the system is dealt with. ENTSO-E ${ }^{2}$ categorizes reserves into three groups. Frequency Containment Reserve (FCR), i.e., primary control, is activated 210 automatically to stabilize the frequency in a matter of seconds. Frequency Restoration Reserve (FRR) is either activated automatically (aFRR), i.e., secondary control, or manually (mFRR), i.e., fast tertiary control, and restores the system frequency by restoring the balance in the control zone, thereby relieving the activated FCR. Finally, Replacement Reserve (RR), i.e., slow tertiary control, can be used to support or relieve 215 the activated FRR [6]. In the developed model the demand for reserves includes an exogenously-determined component in line with current system imbalances (SIs), and an endogenously-determined component to deal with additional SI volumes due to forecast errors of increasing levels of RES generation. The latter is endogenously-determined as it depends on the installed RES capacity, which is decided upon during the optimization 220 and increases with the RES generation target. The sizing of both components is discussed in Section 2.7. Third, a system-wide RES generation target is imposed to ensure that a predefined share of the consumption is covered by RES (2c):

$$
\sum_{i \in \mathbb{I}} p_{i, h}^{\mathrm{inj}}-\sum_{o \in \mathbb{O}} p_{o, h}^{\mathrm{off}}=D_{h}-p_{h}^{\mathrm{ls}}, \quad \forall h \in \mathbb{H},
$$

\footnotetext{
${ }^{2}$ European Network of Transmission System Operators for Electricity.
} 


$$
\begin{aligned}
& \sum_{i \in \mathbb{I}} r_{r, i, h}^{\mathrm{inj}}+\sum_{o \in \mathbb{O}} r_{r, o, h}^{\mathrm{off}}=R_{r}^{\mathrm{ex}}+\sum_{i \in \mathbb{I}}\left(R_{r, i}^{\mathrm{en}} \cdot p_{i}^{\mathrm{inst}, \mathrm{inj}}\right), \quad \forall r \in \mathbb{R}, h \in \mathbb{H}, \\
& \sum_{h \in \mathbb{H}} \sum_{i \in \mathbb{I}} p_{i, h}^{\mathrm{inj}} \geq S^{\mathrm{res}} \cdot \sum_{h \in \mathbb{H}} D_{h} .
\end{aligned}
$$

\subsection{Dispatchable injection and offtake constraints}

Flexibility is provided through cycling, which can be defined as changing the output by starting up, shutting down, or ramping up and down. Techno-economic constraints that limit this cycling include commitment decisions, start-up and shut-down costs, minimum and maximum output levels, minimum up and down times, and ramp rates and costs. Since the modeling of dispatchable injections and offtakes is quite similar, only the constraints for the former are described here. They only differ in the provision of reserve capacity: while a potential increase in injection output contributes to the provision of upward reserve, a potential increase in offtake output contributes to the provision of downward reserve, and vice versa for a potential decrease in output. While the operation of dispatchable generators is fully described by (3a)-(5l), storage operation is additionally subject to the offtake constraints and constraints discussed in Section 2.6.

\subsubsection{Commitment constraints}

A cluster's number of online units can change by starting up offline units or shutting down online units (3a). It is limited to the maximum number of online units, determined by the ratio of the installed capacity and typical unit size (3b). The number of offline units that can start up, or be reserved to start up to provide reserve, is limited to the units that have been offline for at least the minimum down time (3c). Similarly, the number of online units that can shut down, or be reserved to shut down to provide reserve, is limited to the units that have been online for at least the minimum up time (3d):

$$
\begin{array}{ll}
n_{i, h+1}^{\text {inj }}=n_{i, h}^{\text {inj }}+n_{i, h}^{\text {su,inj }}-n_{i, h}^{\text {sd,inj }}, & \forall i \in \mathbb{D}, h \in \mathbb{H}, \\
n_{i, h}^{\text {inj }} \leq p_{i}^{\text {inst,inj }} / P_{i}^{\text {inj }}, & \forall i \in \mathbb{D}, h \in \mathbb{H}, \\
n_{i, h}^{\text {su,inj }}+\sum_{r \in \mathbb{R} \mathbb{U}} n_{r, i, h}^{\text {sur,inj }} \leq p_{i}^{\text {inst,inj }} / P_{i}^{\text {inj }}-n_{i, h}^{\text {inj }}-\sum_{z \in \mathbb{Z}} n_{i, h-z}^{\text {sd,inj }}, & \forall i \in \mathbb{D}, h \in \mathbb{H}, \\
n_{i, h}^{\text {sd,inj }}+\sum_{r \in \mathbb{R} \mathbb{D}} n_{r, i, h}^{\text {sdr,inj }} \leq n_{i, h}^{\text {inj }}-\sum_{w \in \mathbb{W}} n_{i, h-w}^{\text {su,inj }}, & \forall i \in \mathbb{D}, h \in \mathbb{H} .
\end{array}
$$

\subsubsection{Output level constraints}

A cluster's output level can change by ramping online units up or down, starting up offline units, or shutting down online units (4a). The output level is limited by the generation limits of the online units (4b)-(4c). Units starting up have to at least reach the minimum output level, and are constrained by the start-up ramp rate (4d)-(4e). A technology's start-up ramp rate is defined as the maximum of the required ramp rate to reach the minimum output level over one time step and the spinning ramp rate to allow all technologies to start-up in one hourly time step. Similarly, units shutting down have to be able to ramp down to a zero output level from at least the minimum output level, and are constrained by the shut-down ramp rate (4f)- $(4 \mathrm{~g})$, which is defined similar to 
255 the start-up ramp rate. Ramping online units up and down is limited by the spinning ramp rate, while ensuring that ramping ability reserved for reserve provision is accounted for separately from the ramping that occurs in the scheduling phase to provide energy services (4h)-(4i). In addition to the spinning ramp rate, the ramping ability for online units is also constrained by the capacity available to perform spinning ramping $(4 \mathrm{j})-(4 \mathrm{k})$ :

$$
\begin{aligned}
& p_{i, h+1}^{\mathrm{inj}}=p_{i, h}^{\mathrm{inj}}+p_{i, h}^{\mathrm{ru}, \mathrm{inj}}-p_{i, h}^{\mathrm{rd}, \mathrm{inj}}+p_{i, h}^{\mathrm{su}, \mathrm{inj}}-p_{i, h}^{\mathrm{sd}, \mathrm{inj}}, \\
& \forall i \in \mathbb{I D}, h \in \mathbb{H} \text {, } \\
& p_{i, h}^{\mathrm{inj}} \geq n_{i, h}^{\mathrm{inj}} \cdot P_{i}^{\mathrm{min}, \mathrm{inj}} \cdot P_{i}^{\mathrm{inj}}, \\
& p_{i, h}^{\text {inj }} \leq n_{i, h}^{\text {inj }} \cdot P_{i}^{\text {inj }}, \\
& p_{i, h}^{\mathrm{su}, \mathrm{inj}} \geq n_{i, h}^{\mathrm{su}, \mathrm{inj}} \cdot P_{i}^{\mathrm{min}, \mathrm{inj}} \cdot P_{i}^{\mathrm{inj}}, \\
& p_{i, h}^{\mathrm{su}, \mathrm{inj}} \leq n_{i, h}^{\mathrm{su}, \mathrm{inj}} \cdot R_{i}^{\mathrm{su}, \mathrm{inj}} \cdot P_{i}^{\mathrm{inj}}, \\
& p_{i, h}^{\text {sd,inj }} \geq n_{i, h}^{\text {sd,inj }} \cdot P_{i}^{\text {min,inj }} \cdot P_{i}^{\text {inj }} \text {, } \\
& p_{i, h}^{\mathrm{sd}, \mathrm{inj}} \leq n_{i, h}^{\mathrm{sd}, \mathrm{inj}} \cdot R_{i}^{\mathrm{sd}, \mathrm{inj}} \cdot P_{i}^{\mathrm{inj}}, \\
& p_{i, h}^{\mathrm{ru}, \mathrm{inj}}+\sum_{\mathbb{R U}} r_{r, i, h}^{\mathrm{s}, \mathrm{inj}} \leq\left(n_{i, h}^{\mathrm{inj}}-n_{i, h}^{\mathrm{sd}, \mathrm{inj}}\right) \cdot R_{i}^{\mathrm{s}, \mathrm{inj}} \cdot P_{i}^{\mathrm{inj}}, \\
& p_{i, h}^{\mathrm{rd}, \mathrm{inj}}+\sum_{\mathbb{R} \mathbb{D}} r_{r, i, h}^{\mathrm{s}, \mathrm{inj}} \leq\left(n_{i, h}^{\mathrm{inj}}-n_{i, h}^{\mathrm{sd}, \mathrm{inj}}-\sum_{\mathbb{R D}} n_{r, i, h}^{\mathrm{sdr}, \mathrm{inj}}\right) \cdot R_{i}^{\mathrm{s}, \text { inj }} \cdot P_{i}^{\mathrm{inj}}, \\
& p_{i, h}^{\mathrm{ru}, \mathrm{inj}}+\sum_{\mathbb{R} \mathbb{U}} r_{r, i, h}^{\mathrm{s}, \mathrm{inj}} \leq\left(n_{i, h}^{\mathrm{inj}}-n_{i, h}^{\mathrm{sd}, \mathrm{inj}}\right) \cdot P_{i}^{\mathrm{inj}}-\left(p_{i, h}^{\mathrm{inj}}-p_{i, h}^{\mathrm{sd}, \mathrm{inj}}\right), \\
& p_{i, h}^{\mathrm{rd}, \mathrm{inj}}+\sum_{\mathbb{R} \mathbb{D}} r_{r, i, h}^{\mathrm{s}, \mathrm{inj}} \leq\left(p_{i, h}^{\mathrm{inj}}-p_{i, h}^{\mathrm{sd}, \mathrm{inj}}-\sum_{\mathbb{R} \mathbb{D}} r_{r, i, h}^{\mathrm{sd}, \mathrm{inj}}\right) \\
& -\left(n_{i, h}^{\mathrm{inj}}-n_{i, h}^{\mathrm{sd}, \mathrm{inj}}-\sum_{\mathbb{R D}} n_{r, i, h}^{\mathrm{sdr}, \mathrm{inj}}\right) \cdot P_{i}^{\mathrm{min}, \mathrm{inj}} \cdot P_{i}^{\mathrm{inj}}, \\
& \forall i \in \mathbb{I D}, h \in \mathbb{H} \text {, } \\
& \forall i \in \mathbb{I D}, h \in \mathbb{H} \text {, } \\
& \forall i \in \mathbb{I D}, h \in \mathbb{H} \text {, } \\
& \forall i \in \mathbb{I D}, h \in \mathbb{H} \text {, } \\
& \forall i \in \mathbb{I D}, h \in \mathbb{H} \text {, } \\
& \forall i \in \mathbb{I D}, h \in \mathbb{H} \text {, } \\
& \forall i \in \mathbb{I D}, h \in \mathbb{H} \text {, }
\end{aligned}
$$

\subsubsection{Reserve provision constraints}

Dispatchable injection technologies provide upward reserve through online units that can increase their output and offline units that can start up (5a), and downward reserve through online units that can decrease their output or shut down (5b). Contracting FCR with injection technology $i$ is limited by the technology's FCR-specific spinning ramp rate $(5 \mathrm{c}),(5 \mathrm{f})$, while contracting FCR plus aFRR is limited by the aFRR-specific spinning ramp rate $(5 \mathrm{~d}),(5 \mathrm{~g})$, and contracting FCR plus aFRR plus mFRR is limited by the mFRR-specific spinning ramp rate (5e), (5h). Units providing reserve through starting up or shutting down are also limited by the technology's ramping ability, and need to be able to overcome at least the minimum output level (5i)-(5l):

$$
\begin{aligned}
& r_{r, i, h}^{\mathrm{inj}}=r_{r, i, h}^{\mathrm{s}, \mathrm{inj}}+r_{r, i, h}^{\mathrm{su}, \mathrm{inj}}, \\
& \forall r \in \mathbb{R U}, i \in \mathbb{I D}, h \in \mathbb{H},(5 \mathrm{a}) \\
& r_{r, i, h}^{\mathrm{inj}}=r_{r, i, h}^{\mathrm{s}, \mathrm{inj}}+r_{r, i, h}^{\mathrm{sd}, \mathrm{inj}}, \\
& \forall r \in \mathbb{R D}, i \in \mathbb{I D}, h \in \mathbb{H} \text {, } \\
& \sum_{r \in \mathbb{R} U \mathbb{F}} r_{r, i, h}^{\mathrm{s}, \mathrm{inj}} \leq\left(n_{i, h}^{\mathrm{inj}}-n_{i, h}^{\mathrm{sd}, \mathrm{inj}}\right) \cdot R_{f c r, i}^{\mathrm{s}, \mathrm{r}, \mathrm{inj}} \cdot P_{i}^{\mathrm{inj}}, \\
& \forall i \in \mathbb{I D}, h \in \mathbb{H} \text {, } \\
& \sum_{r \in \mathbb{R U A}} r_{r, i, h}^{\mathrm{s}, \mathrm{inj}} \leq\left(n_{i, h}^{\mathrm{inj}}-n_{i, h}^{\mathrm{sd}, \mathrm{inj}}\right) \cdot R_{a f r r, i}^{\mathrm{s}, \mathrm{r}, \mathrm{inj}} \cdot P_{i}^{\mathrm{inj}}, \\
& \forall i \in \mathbb{I D}, h \in \mathbb{H} \text {, }
\end{aligned}
$$




$$
\begin{aligned}
& \sum_{r \in \mathbb{R} \mathbb{U}} r_{r, i, h}^{\mathrm{s}, \mathrm{inj}} \leq\left(n_{i, h}^{\mathrm{inj}}-n_{i, h}^{\mathrm{sd}, \mathrm{inj}}\right) \cdot R_{m f r r, i}^{\mathrm{s}, \mathrm{r}, \mathrm{inj}} \cdot P_{i}^{\mathrm{inj}}, \quad \forall i \in \mathbb{I D}, h \in \mathbb{H}, \\
& \sum_{r \in \mathbb{R} \mathbb{D F}} r_{r, i, h}^{\mathrm{s}, \mathrm{inj}} \leq\left(n_{i, h}^{\mathrm{inj}}-n_{i, h}^{\mathrm{sd}, \mathrm{inj}}-\sum_{r \in \mathbb{R} \mathbb{D}} n_{r, i, h}^{\mathrm{sdr}, \mathrm{inj}}\right) \cdot R_{f c r, i}^{\mathrm{s}, \mathrm{r}, \mathrm{inj}} \cdot P_{i}^{\mathrm{inj}}, \quad \forall i \in \mathbb{I} \mathbb{D}, h \in \mathbb{H}, \\
& \sum_{r \in \mathbb{R D A} \mathbb{A}} r_{r, i, h}^{\mathrm{s}, \mathrm{inj}} \leq\left(n_{i, h}^{\mathrm{inj}}-n_{i, h}^{\mathrm{sd}, \mathrm{inj}}-\sum_{r \in \mathbb{R} \mathbb{D}} n_{r, i, h}^{\mathrm{sdr}, \mathrm{inj}}\right) \cdot R_{a f r r, i}^{\mathrm{s}, \mathrm{r}, \mathrm{inj}} \cdot P_{i}^{\mathrm{inj}}, \quad \forall i \in \mathbb{I D}, h \in \mathbb{H}, \\
& \sum_{r \in \mathbb{R} \mathbb{D}} r_{r, i, h}^{\mathrm{s}, \mathrm{inj}} \leq\left(n_{i, h}^{\mathrm{inj}}-n_{i, h}^{\mathrm{sd}, \mathrm{inj}}-\sum_{r \in \mathbb{R} \mathbb{D}} n_{r, i, h}^{\mathrm{sdr}, \mathrm{inj}}\right) \cdot R_{m f r r, i}^{\mathrm{s}, \mathrm{r}, \mathrm{inj}} \cdot P_{i}^{\mathrm{inj}}, \quad \forall i \in \mathbb{I D}, h \in \mathbb{H}, \\
& r_{r, i, h}^{\mathrm{su}, \mathrm{inj}} \geq n_{r, i, h}^{\mathrm{sur}, \mathrm{inj}} \cdot P_{i}^{\mathrm{min}, \mathrm{inj}} \cdot P_{i}^{\mathrm{inj}}, \\
& r_{r, i, h}^{\mathrm{su}, \mathrm{inj}} \leq n_{r, i, h}^{\mathrm{sur}, \mathrm{inj}} \cdot R_{r, i}^{\mathrm{s}, \mathrm{r}, \mathrm{inj}} \cdot P_{i}^{\mathrm{inj}}, \\
& r_{r, i, h}^{\mathrm{sd}, \mathrm{inj}} \geq n_{r, i, h}^{\mathrm{sdr}, \mathrm{inj}} \cdot P_{i}^{\mathrm{min}, \mathrm{inj}} \cdot P_{i}^{\mathrm{inj}}, \\
& r_{r, i, h}^{\mathrm{sd}, \text { inj }} \leq n_{r, i, h}^{\mathrm{sdr}, \text { inj }} \cdot R_{r, i}^{\mathrm{s}, \mathrm{r}, \mathrm{inj}} \cdot P_{i}^{\mathrm{inj}},
\end{aligned}
$$

\subsection{Intermittent injection constraints}

Renewable generation volumes are driven by weather conditions and support schemes, rather than by electricity prices. As such, it is usually modeled as negative load, resulting in a residual load to be met by dispatchable units. However, the renewable generators participation in electricity markets is becoming increasingly active, with the possibility to curtail output. They have close-to-zero (or even negative if subsidized) marginal costs, a time-varying maximum power output, and limited operational constraints. The timevarying RES output is calculated by using a normalized feed-in profile, which is scaled with the installed capacity. This available output can either be injected in the grid to be consumed, or curtailed in case of oversupply (6):

$$
p_{i, h}^{\mathrm{inj}}+p_{i, h}^{\mathrm{cu}, \mathrm{inj}}=A_{i, h}^{\mathrm{res}} \cdot p_{i}^{\mathrm{inst}, \mathrm{inj}}, \quad \forall i \in \mathbb{I I}, h \in \mathbb{H} .
$$

Although RES may provide contracted reserve to the TSO if tender periods are sufficiently short (e.g., hours), they are not able to contribute in the provision of reserve in the analyzed case study due to the assumed monthly contract periods (Section 2.7). In current markets especially wind generators already provide downward reserve through non-contracted reserve for short periods of time. In the future, these may provide upward reserve as well when constantly performing under their availability limit, and PV systems may also provide reserve through improved control and aggregation.

\subsection{Electricity storage constraints}

290

Storage systems are subject to energy buffer dynamics and a limited cycle-life. Furthermore, a symmetrical development of charge and discharge power ratings is assumed.

During charging, only part of the consumed electric energy is converted to energy stored in the buffer due to a charge efficiency, while during discharging, only part of the stored energy is converted back into electric energy due to a discharge efficiency (7a).

295 These additions and removals have to respect the minimum and maximum storage capacity, while the available range to provide energy services is constrained in both directions by the margins that are contracted for reserve provision $(7 b)-(7 \mathrm{c})$. Linear ramping is 
assumed from the current output level to the output after activation in $T_{r}^{1, \mathrm{r}}$. The energy capacity that is reserved for reserve provision is assumed to be the energy required for the linear ramping and to maintain reserve provision up to $T_{r}^{2, \mathrm{r}}$ (Fig. 1).

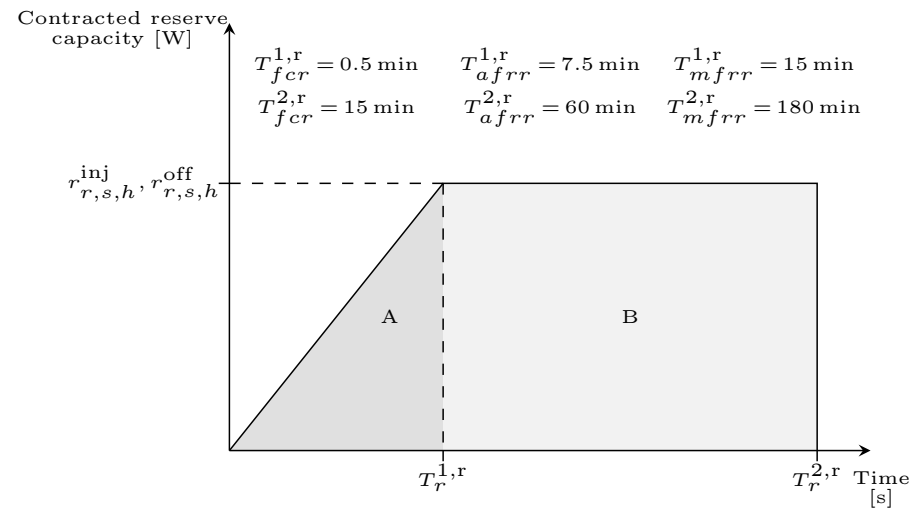

Figure 1: Energy storage capacity that needs to be reserved to provide reserve capacity $(A+B)$.

Storage plants have a limited lifetime, which is either determined by the calendar life in case of infrequent use or by the cycle-life in case of frequent use. The calendar life is the maximum time that it can be used, independent from the operation, while the cycle-life takes into account the deterioration of the energy storage subsystem due to use [5, 42].

305 While the cycle-life limits the operation of BES, for PHS the cycle-life is sufficiently large such that the depreciation cost following cycling patterns is negligible. Although there is no direct constraint on the number of cycles during the considered optimization period, due to the limited cycle-life a constant targeted cycling rate is implied throughout the lifetime. If the cycling rate is lower than or equal to this targeted cycling rate, the additional depreciation cost from cycling is zero, otherwise it is positive (7d):

$$
\begin{aligned}
& e_{s, h+1}=e_{s, h}+\left(\eta_{s}^{\mathrm{off}} \cdot p_{s, h}^{\mathrm{off}}-p_{s, h}^{\mathrm{inj}} / \eta_{s}^{\mathrm{inj}}\right) \cdot T^{\mathrm{h}}, \\
& \forall s \in \mathbb{S}, h \in \mathbb{H}, \\
& e_{s, h} \geq\left(1 / \eta_{s}^{\mathrm{inj}}\right) \cdot \sum_{r \in \mathbb{R U}}\left[\left(r_{r, s, h}^{\mathrm{inj}} \cdot T_{r}^{1, \mathrm{r}}\right) / 2+r_{r, s, h}^{\mathrm{inj}} \cdot\left(T_{r}^{2, \mathrm{r}}-T_{r}^{1, \mathrm{r}}\right)\right], \\
& \forall s \in \mathbb{S}, h \in \mathbb{H}, \\
& e_{s, h} \leq e_{s}^{\text {inst }}-\eta_{s}^{\text {off }} \cdot \sum_{r \in \mathbb{R D}}\left[\left(r_{r, s, h}^{\text {off }} \cdot T_{r}^{1, \mathrm{r}}\right) / 2+r_{r, s, h}^{\text {off }} \cdot\left(T_{r}^{2, \mathrm{r}}-T_{r}^{1, \mathrm{r}}\right)\right], \quad \forall s \in \mathbb{S}, h \in \mathbb{H}, \\
& c_{s}^{\text {cyc }} \geq C_{s}^{\text {inv }, \mathrm{e}} \cdot\left(\eta_{s}^{\text {off }} \cdot \sum_{h \in \mathbb{H}} p_{s, h}^{\text {off }} / N_{s}^{\text {cyc }}-e_{s}^{\text {inst }} / N_{s}^{\text {cal,inj }}\right), \quad \forall s \in \mathbb{S} \text {. }
\end{aligned}
$$

\subsection{Reserve sizing}

\subsubsection{FCR sizing}

The required FCR is determined on the ENTSO-E level, and is set at $3 \mathrm{GW}$ for the synchronous area of continental Europe, covering the outage of two of the largest elements (i.e., an N-2 criterion). This effort is shared over the different control zones according to their share in the system $[43,44]$. In line with the volume currently contracted by Elia, an exogenously-determined FCR requirement of $100 \mathrm{MW}$ in both the up- and downward 
direction is included in the analyzed case study. Since Elia does not expect significant changes in the need for FCR [45], the endogenously-determined FCR requirement is zero.

\subsubsection{FRR and RR sizing}

The sizing of FRR and RR is the responsibility of the TSO, subject to ENTSO$\mathrm{E}$ guidelines, and is based on both a deterministic and probabilistic assessment. The deterministic assessment considers the largest possible SI due to the loss of a single grid element. For Belgium the loss of a $1 \mathrm{GW}$ interconnector is considered (i.e., the future Nemo interconnector). The FRR to be contracted has to at least be sufficient to cover such an event in both directions. The probabilistic assessment is based on recent historical SI time series of at least a full year, and determines the combined amount of FRR and RR to be contracted. ENTSO-E requests that the contracted amount of FRR and RR at 330 least should be able to cover $99 \%$ of the observed SIs in both directions (Fig. 2a), which is also imposed in the considered case study. In case the reserve sizing based on the probabilistic assessment results in lower reserve needs than the deterministic assessment, the latter is kept as a minimum for the amount of FRR that needs to be contracted. Although RR may be contracted to cover the gap between both in case the probabilistic 335 assessment results in higher reserve needs than the deterministic analysis, this gap may also be covered by FRR as contracting RR capacity is not required. Since Elia does not contract RR, it is not considered here [43, 44]. Similar to the approach used by Elia [43], after having determined the total FRR need, a time series of the difference between the SI of consecutive quarter-hourly periods, representing the volatility of the SI, is considered

340 to determine the share of aFRR (Fig. 2b). The aFRR to be contracted is determined by the required capacity to cover a certain percentage of the volatility of the SI in both directions. In the analyzed case study this percentage is assumed to be $80 \%$, in line with information provided by Elia [45]. The remaining FRR to be contracted to cover the total FRR need determines the amount of mFRR (Fig. 2c).

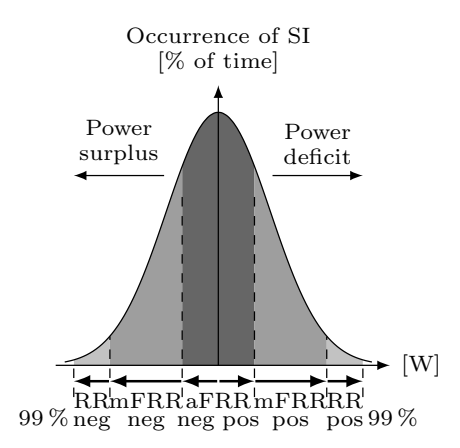

(a) Probabilistic sizing.

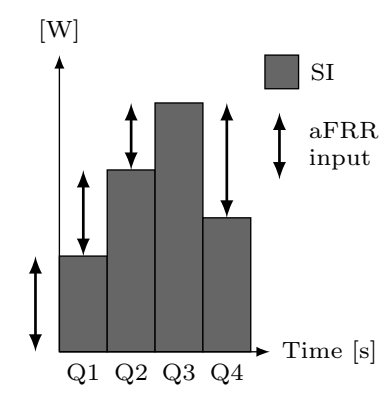

(b) SI volatility.

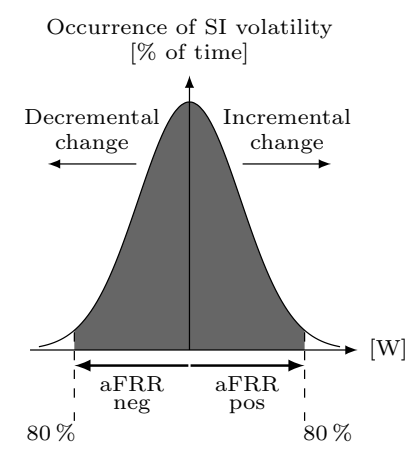

(c) Sizing of aFRR.

Figure 2: Probabilistic reserve sizing in line with the approach used by Elia.

Since the previously discussed sizing determines the FRR need for the current situation, it represents the exogenously-determined aFRR and mFRR requirement. In contrast to FCR, the endogenously-determined aFRR and mFRR requirement is nonzero due to increasing absolute levels of forecast errors with larger RES penetrations. For 
each intermittent RES technology a probability density function (pdf) of the normalized forecast errors is introduced by comparing the day-ahead forecast with the real-time output and describing the error by means of a normal distribution. Similar to the method for the exogenous component, the $99 \%$ quantile in both directions determines the total endogenous FRR requirement. Afterwards, this total FRR requirement is again translated to endogenously-determined aFRR and mFRR needs. Again, the aFRR to be contracted 355 is determined by the required capacity to cover $80 \%$ of the forecast error's volatility. This is then complemented by mFRR to meet the total endogenously-determined FRR needs. It is assumed that variable RES only increase the need for upward reserve. In case of unexpected excess generation, the market design is expected to incentivize RES to curtail output if insufficient alternative downward flexibility providers are available.

\subsubsection{Sizing and contract periods}

In the analyzed case study the reserve requirements are sized on a yearly basis, while, in line with current reserve procurement trends in Europe, shorter contract periods (here monthly) for reserve capacity are considered. Abstraction is made from which share of the reserve requirements is to be procured by the TSO as an ancillary service to balance

365 its control zone, or by market participants to keep their portfolio balanced.

\section{Data, scenarios, and assumptions}

\subsection{Data}

Four dispatchable generation technologies are taken into account, i.e., base, mid, peak, and high peak load, each having different techno-economic characteristics, and ordered in terms of decreasing fixed cost and increasing variable cost. The first two technologies are nuclear and coal power plants, respectively, whereas peak and high peak load technologies correspond to combined cycle gas turbines (CCGTs) and open cycle gas turbines (OCGTs), respectively. In addition, three variable RES technologies, i.e., onshore wind, offshore wind, and PV, and two electricity storage technologies, i.e., PHS and Li-ion BES, are considered. Although many different estimates for the cost data and technical parameters is available, the assumed input data is inspired by [10, 46, 47, 48], and is shown in Table 4 and Table 5 . These values may deviate from actual levels, but the relative levels for the different technologies are believed to be representative.

To limit the reserve capacity that can be provided, the ramp rate on a minute basis $R_{i}^{\mathrm{m}, \mathrm{inj}} / R_{o}^{\mathrm{m}, \text { off }}$ is used. In line with guidelines from the Belgian TSO Elia [49], we assume that capacity providing reserve has to be able to perform the promised change in power output in $0.5 \mathrm{~min}$ for FCR, $7.5 \mathrm{~min}$ for aFRR, and $15 \mathrm{~min}$ for $\mathrm{mFRR}$. As such, the technologies' spinning ramp rate for each reserve category $R_{r, i}^{\mathrm{s}, \mathrm{r}, \mathrm{inj}} / R_{r, o}^{\mathrm{s}, \mathrm{r} \text { off }}$ can be derived. Since the ramp rate on a minute basis is usually defined as being faster compared to 385 the hourly ramp rate, the reserve-specific spinning ramp rate is limited by the hourly ramp rate to avoid situations in which more ramping is possible in $7.5 \mathrm{~min}$ or $15 \mathrm{~min}$ than in an hour. While those fast ramp rates on a minute basis may be appropriate for infrequent use (i.e., reserve provision), they are believed to be too high for continuous operation (i.e., electricity generation), potentially incurring additional O\&M costs. As

390 such, the hourly ramp rates are used for ramping in the scheduling phase to provide energy services. 
Table 4: Economic input parameters, fixed costs are annualized via annuities using a $5 \%$ interest rate. Electricity storage charge and discharge parameters are assumed to be identical.

\begin{tabular}{|c|c|c|c|c|c|c|c|c|c|c|c|}
\hline \multirow[b]{3}{*}{ Name } & \multirow[b]{3}{*}{$\begin{array}{c}N_{i}^{\text {cal, inj }} \\
{[\mathrm{a}]}\end{array}$} & \multicolumn{5}{|c|}{ Fixed costs } & \multicolumn{5}{|c|}{ Variable costs } \\
\hline & & \multicolumn{2}{|c|}{ Total } & \multicolumn{3}{|c|}{ Annualized } & \multirow[b]{2}{*}{$\begin{array}{c}C_{i}^{\text {fuel,inj }} \\
{[€ / \mathrm{MWh}]}\end{array}$} & \multirow[b]{2}{*}{$\begin{array}{c}C_{i}^{\text {vom,inj }} \\
{[€ / \mathrm{MWh}]}\end{array}$} & \multirow[b]{2}{*}{$\begin{array}{c}C_{i}^{\mathrm{ra}, \mathrm{inj}} \\
{[€ / \mathrm{MW}]} \\
\end{array}$} & \multirow[b]{2}{*}{$\begin{array}{c}C_{i}^{\mathrm{su}, \mathrm{inj}} \\
{[€ / \mathrm{MW}]}\end{array}$} & \multirow[b]{2}{*}{$\begin{array}{c}C_{i}^{\text {sd,inj }} \\
{[€ / \mathrm{MW}]}\end{array}$} \\
\hline & & $\begin{array}{l}C_{i}^{\text {inv,inj }} \\
{[€ / \mathrm{kW}]}\end{array}$ & $\begin{array}{c}C_{s}^{\text {inv,e }} \\
{[€ / \mathrm{kWh}]} \\
\end{array}$ & $\begin{array}{c}C_{i}^{\text {inv,inj }} \\
{[€ / \mathrm{kW}]}\end{array}$ & $\begin{array}{c}C_{s}^{\text {inv,e }} \\
{[€ / \mathrm{kWh}]}\end{array}$ & $\begin{array}{l}C_{i}^{\text {tom,inj }} \\
{[€ / \mathrm{kW}]}\end{array}$ & & & & & \\
\hline Base & 50 & 5000 & - & 274 & - & 43 & 10 & 5 & 1.30 & 200 & 0 \\
\hline Mid & 35 & 1700 & - & 104 & - & 34 & 26 & 10 & 1.30 & 50 & 0 \\
\hline Peak & 25 & 855 & - & 61 & - & 21 & 43 & 10 & 0.7 & 37 & 0 \\
\hline hPeak & 15 & 486 & - & 47 & - & 12 & 66 & 10 & 0.3 & 25 & 0 \\
\hline PV & 25 & 895 & - & 64 & - & 13 & - & 0 & - & - & - \\
\hline onWind & 30 & 1270 & - & 83 & - & 27 & - & 0 & - & - & - \\
\hline ofWind & 30 & 2600 & - & 169 & - & 80 & - & 0 & - & - & - \\
\hline BES & 15 & 100 & 300 & 10 & 29 & 0 & - & 0 & 0 & 0 & 0 \\
\hline PHS & 50 & 375 & 50 & 21 & 3 & 0 & - & 0 & 0 & 0 & 0 \\
\hline
\end{tabular}

Table 5: Technical input parameters. Electricity storage charge and discharge parameters are assumed to be identical, except for the minimum load requirement $\left(P_{i}^{\mathrm{min}, \mathrm{inj}} / P_{o}^{\mathrm{min}, \mathrm{off}}\right)$.

\begin{tabular}{|c|c|c|c|c|c|c|c|c|}
\hline Name & $\begin{array}{c}P_{i}^{\mathrm{inj}} \\
{[\mathrm{MW}]}\end{array}$ & $\begin{array}{c}P_{i}^{\min , \text { inj }} \\
{[\%]}\end{array}$ & $\begin{array}{c}R_{i}^{\mathrm{s}, \mathrm{inj}} \\
{[\%]}\end{array}$ & $\begin{array}{c}R_{i}^{\mathrm{m}, \mathrm{inj}} \\
{[\% / \mathrm{min}]}\end{array}$ & $\begin{array}{l}\eta_{s}^{\mathrm{inj}} \\
{[\%]}\end{array}$ & $\begin{array}{c}T_{i}^{\text {mut,inj }} \\
\quad[h]\end{array}$ & $\begin{array}{l}T_{i}^{\text {mdt,inj }} \\
\quad[\mathrm{h}]\end{array}$ & $\begin{array}{c}N_{s}^{c y c} \\
{[-]}\end{array}$ \\
\hline Base & 400 & 50 & 33 & 3 & - & 24 & 24 & - \\
\hline Mid & 300 & 50 & 50 & 4 & - & 6 & 4 & - \\
\hline Peak & 200 & 50 & 80 & 6 & - & 4 & 1 & - \\
\hline hPeak & 100 & 10 & 100 & 10 & - & 1 & 1 & - \\
\hline BES & 10 & 0 & 100 & 100 & 95 & 0 & 0 & 3000 \\
\hline PHS & 100 & $30 / 70$ & 100 & 50 & 87 & 0 & 0 & $\infty$ \\
\hline
\end{tabular}

We use historical RES power output data from Elia [50] and consumption data from ENTSO-E [51], on an hourly basis, for Belgium for 2014. For this period PV is characterized by 1054 full load hours, while onshore and offshore wind are characterized by 2046 and 3600 full load hours, respectively. Average consumption is $9539 \mathrm{MW}$, fluctuating between a minimum of $6623 \mathrm{MW}$ and a maximum of $13110 \mathrm{MW}$. Using data from Elia, the exogenous aFRR and mFRR requirements are $157 \mathrm{MW}$ and $843 \mathrm{MW}$, respectively, in both directions, while the endogenous aFRR and $\mathrm{mFRR}$ requirements amount to $0.01 \mathrm{MW}$ and $0.12 \mathrm{MW}$ per MW PV, $0.02 \mathrm{MW}$ and $0.15 \mathrm{MW}$ per MW onshore wind, and $0.05 \mathrm{MW}$ and 0.33 MW per MW offshore wind, respectively, all in the upward direction.

Since a high RES curtailment cost corresponds to today's electricity markets with subsidies, and a zero (or low) RES curtailment cost corresponds to future markets without subsidies but with active RES participation, we assume a RES curtailment cost of $0 € /$ MWh. Finally, the cost of involuntary load shedding is set at $3000 € / \mathrm{MWh}$, based on the price cap in the day-ahead market of the Central Western European region.

\subsection{Scenarios}

The portfolio and operation of the system is optimized with an hourly time resolution. Five increasing targets for the share of RES in the final consumption, ranging from $0 \%$ to $50 \%$, are considered. Furthermore, four storage scenarios are considered. The reference storage scenario, in which no storage is available to be installed, serves as benchmark. By comparing it with the results of the other three scenarios, the role and value of electricity storage can be analyzed. In scenario 1 both PHS and BES is available to be installed, while in scenario 2 the available PHS energy capacity is limited to $8.7 \mathrm{GWh}$. The chosen upper limit is based on the conventional Belgian PHS capacity, considering 
415 the currently developed capacity, i.e., Coo-Trois-Ponts I and II, and Plate-Taille, and the recently proposed extension of the Coo-Trois-Ponts PHS plant [3]. Finally, scenario 3 studies the impact of a future reduction of the energy-related investment cost of BES from $300 € / \mathrm{kWh}$ to $150 € / \mathrm{kWh}$, while keeping the upper limit for PHS at $8.7 \mathrm{GWh}$.

\subsection{Assumptions}

420

First, although the test system includes system load and RES generation characteristics for Belgium, we abstract from an actual system with capacity legacy but instead adopt a long-term greenfield approach. While this does not allow us to derive deployment scenarios or optimal pathways, it allows for broadly applicable system-independent insights in the role and value of storage technologies and in the interdependency of the

425 included flexibility options. The developed GEP model however allows to include capacity legacy by imposing starting values per technology, and can thus also be used for studies that focus on optimal future portfolios for specific countries or regions.

Second, since not all services that storage can provide are considered (e.g., voltage support, congestion management, and black-start capabilities), this analysis may underestimate the total value of storage for the system.

Third, since the different flexibility sources are to some extent interchangeable, the transition to a RES-based power system can be achieved through various portfolios of flexibility sources. As flexible demand is not considered, the results may overestimate the absolute supply of flexibility by storage. In addition, since exchange with neighboring

435 regions is not considered, the possibility to import flexibility supply or to smoothen system variability is neglected, thereby most likely overestimating the need for local flexibility. Furthermore, the linear scaling of historic RES generation profiles further overestimates absolute flexibility needs, as it neglects possibly smoother RES generation profiles by future changes in geographical distribution.

Fourth, in GEP it is common practice to ignore the internal grid, to not constrain the applicability of the results by the current network. We assume a copper plate because the grid can be upgraded in the long-run and we aim to derive broadly applicable insights, and in order to be able to solve numerically for a full year with high operational detail. Instead, GEP is typically considered separately from network expansion planning (NEP), with the GEP output serving as input for the NEP model. We thus do not distinguish between the locations or voltage levels to which installed generation and storage capacities are connected. In real systems the total flexibility need may consist of needs at the transmission and distribution level, possibly requiring different technical solutions.

Fifth, the various sources of uncertainty (e.g., load, RES generation) are addressed with a deterministic approach, given the computational complexity due to the included high temporal and operational detail. This is in line with other GEP works including such detailed short-term operation, as for these models it is computationally impractible to consider the recent developments in stochastic UC formulations. Decisions are based on expected values of probabilistic input parameters, but three reserve products are contracted and scheduled to deal with deviations from these expected values, and thus to deal with uncertainty. The reserve sizing includes a deterministic and probabilistic assessment, and considers unexpected outages and unexpected variations in the load and RES generation. It provides both exogenous and endogenous reserve requirements. The latter increase with the installed variable RES capacity to deal with the increasing uncertainty in the system due to its limited predictability. 
These simplifications contribute to the computational solvability of the presented combined long-term investment and detailed short-term operation model for a full year, and to the traceability of results. We aim to derive general conclusions on the role and value of electricity storage in renewable power systems, thereby not focusing on absolute numbers in the results of individual scenarios, but on orders of magnitude and differences between the four scenarios.

\section{Results}

\subsection{Total system cost}

The availability of electricity storage lowers total system cost (Fig. 3). This is true for all three storage scenarios, and its explanation is threefold. First, storage can compensate the system's expected variability by storing base load and RES generation in times of low residual load, and (partly) replacing peak and high peak load generation in times of high residual load. As such, the rather inflexible base and mid load generation technologies can be operated more efficiently, while the need for flexible peak and high peak load

475 generators decreases. The negative correlation among RES penetration and storage fuel cost, the latter being related to the efficiency losses and the price at which energy is stored, represents a valuable benefit for storage that increases with the RES target. Second, storage can compensate the system's unexpected variability by providing reserve. As such, inefficient scheduling to keep conventional generators online (partly-loaded) to

480 provide spinning reserve can be reduced. ${ }^{3}$ This lowers the incompressible part of supply, thereby decreasing RES curtailment in times of low residual demand, and contributes to efficient merit-order scheduling. Third, less RES capacity needs to be installed to reach the imposed RES targets. Excess RES generation that otherwise had to be curtailed can now be stored, or simply generated to be consumed since the incompressible part of 485 supply is lower with storage as flexibility provider compared to conventional generators.

Scenario 1 leads to the lowest system cost, while scenario 2 is still characterized by significant cost savings compared to the reference scenario but ends up at a higher total cost than scenario 1 . Finally, in scenario 3, the total system cost decreases again compared to scenario 2 but remains well above scenario 1 .

\section{4.2. Generation and storage mix}

Fig. 4 shows the installed capacities for the reference scenario and the three storage scenarios, based on which four observations can be made. First, when storage resources are available, less RES capacity is needed to reach the imposed RES target. Depending on the storage scenario, this leads to $9.7 \%-10.4 \%, 10.8 \%-16.7 \%$, and $9.5 \%-17.0 \%$ less installed RES capacity to reach a $30 \%, 40 \%$, and $50 \%$ RES target, respectively. This may be important in countries where the available land area for wind turbines or PV systems is scarce or faces opposition. Second, storage resources allow base load plants to remain in the optimal mix to a larger extent. Depending on the storage scenario, its installed capacity increases by $67.2 \%-67.9 \%, 76.2 \%-88.7 \%$, and $244.0 \%-369.3 \%$ for

\footnotetext{
${ }^{3}$ Upward FCR and aFRR is provided as spinning reserve by base, mid, and peak generators as they cannot start-up in time to provide them as non-spinning reserve. In addition, all downward reserve categories are provided as spinning reserve by all conventional generators.
} 


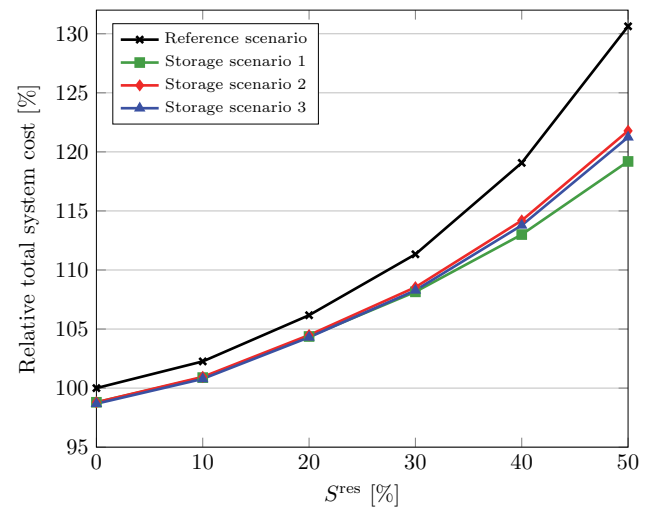

Figure 3: Total system cost relative to the case with a $0 \%$ RES target in the reference scenario.

500 a $0 \%, 10 \%$, and $20 \%$ RES target, respectively. In addition, in scenario 1 base load is even included (to a very limited extent) up to a $30 \%$ RES target compared to only a $20 \%$ RES target for the other scenarios. Third, storage reduces the need for peak and high peak generators. Depending on the storage scenario, the installation of such power plants decreases by $43.1 \%-62.6 \%, 38.7 \%-74.4 \%$, and $36.0 \%-76.6 \%$ for a $30 \%$,

$50540 \%$, and $50 \%$ RES target, respectively. The impact of observations one to three is always the largest in storage scenario 1 and the smallest in scenario 2, with the impact in scenario 3 in between. Fourth, when the maximum available PHS energy capacity is limited due to geographical constraints, PHS is mainly replaced by peak and high peak generation capacity, and only to a limited extent by BES at the current energy-related BES investment cost. At a future lower investment cost of the BES energy storage subsystem again less peak and high peak generation is needed while the installed BES power rating even surpasses PHS from a $30 \%$ RES target onwards.

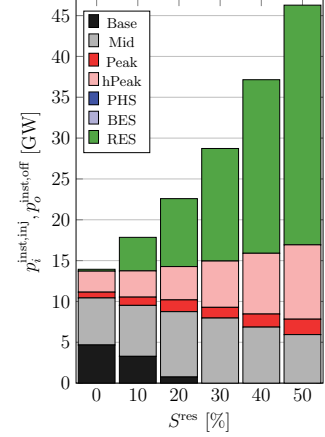

(a) Reference scenario.

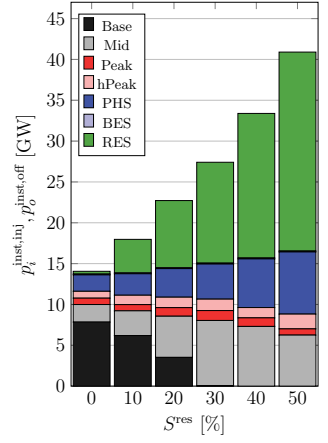

(b) Storage scenario 1.

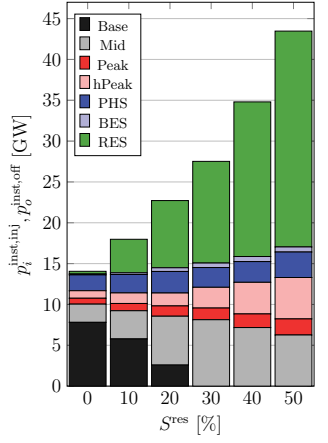

(c) Storage scenario 2 .

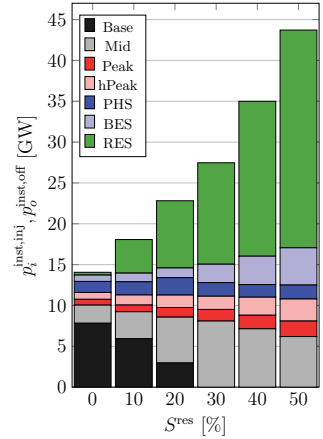

(d) Storage scenario 3.

Figure 4: Optimal mix in different storage scenarios given a variety of RES targets.

Fig. 5 shows that a relationship between the imposed RES target and installed flexible resources (i.e., peak and high peak generation, PHS, and BES) can be observed independent from the analyzed scenario. This may represent the flexibility need at different RES 
targets, met by the different flexibility sources. Although no absolute numbers can be concluded upon since this is most likely dependent on the residual load profile, it shows that flexibility sources are to some extent interchangeable. This is important for regulators and policy-makers to take into account, e.g., when deciding on capacity markets, as these generally result in current gas-fired conventional generators being contracted to remain operational (e.g., strategic reserve in Belgium, capacity auction in the UK). As such, this may constrain the development of alternative flexibility sources.

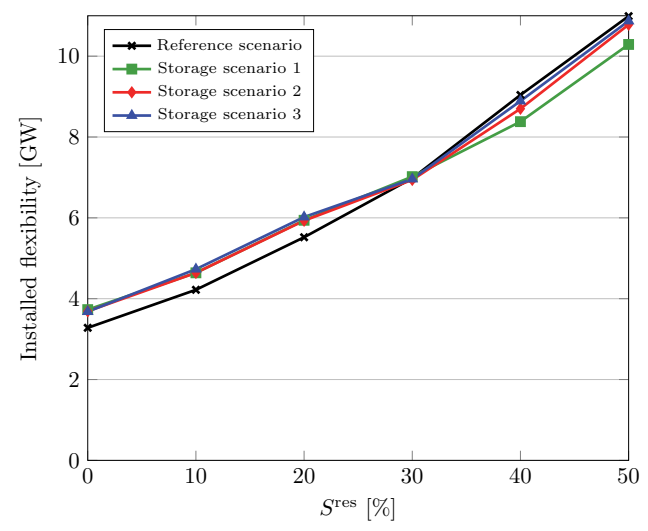

Figure 5: Relationship between the installed flexible capacity and imposed RES target.

Finally, an analysis of the energy, power, and E2P ratio characteristics of the installed storage resources in the three storage scenarios is provided in Table 6. First, in scenario 1 a significant amount of PHS is developed, both in terms of energy and power, which includes an E2P ratio between $4.56 \mathrm{~h}$ and $8.39 \mathrm{~h}$. The developed PHS is used for both energy-related and power-related services. In contrast, BES energy capacity and power rating is only developed to a limited extent, with the former being small compared to the latter. The resulting E2P ratio is between $0.24 \mathrm{~h}$ and $0.47 \mathrm{~h}$, as BES is almost exclusively used to provide power-related frequency control in this scenario. Second, in scenario 2 the total available PHS energy capacity is immediately developed from a $0 \%$ RES target, while the accompanying installed PHS power rating increases moderately with the RES target. This leads to E2P ratios between $2.80 \mathrm{~h}$ and $4.49 \mathrm{~h}$. Scenario 2 includes both higher BES energy capacity and power rating levels compared to scenario 535 1, but E2P ratios have similar orders of magnitude (i.e., $0.25 \mathrm{~h}-0.82 \mathrm{~h}$ ). While BES takes over part of the power-related services of PHS, flexible generators cover its energyrelated services. Third, scenario 3 shows that the available PHS energy capacity is fully developed from the start even at a lower energy-related BES investment cost, but less power rating is developed. This leads to higher E2P ratios for PHS compared to scenario

540 2, i.e., $4.09 \mathrm{~h}-6.43 \mathrm{~h}$. Significantly more BES energy capacity is developed, surpassing the maximum available PHS energy capacity at a $50 \%$ RES target, as well as more power rating, surpassing the installed PHS power rating at high RES targets. Although the BES E2P ratio increases to $1.10 \mathrm{~h}-2.26 \mathrm{~h}$, it is still well below the PHS E2P ratio. This analysis shows that both short-to-mid and mid-to-long-term storage is needed: even

${ }_{545}$ when PHS would be available to an unlimited extent, BES is developed, and even when the energy-related BES investment cost would decrease towards the future, the available 
PHS energy capacity is still fully developed. Although these sources compete to provide some flexibility services, they complement each other to meet the system's total flexibility demand in the most efficient way.

Table 6: Storage characteristics in the different storage scenarios at different RES targets.

\begin{tabular}{|c|c|c|c|c|c|c|c|c|c|c|c|c|c|c|}
\hline & & & \multicolumn{6}{|c|}{ PHS } & \multicolumn{6}{|c|}{ BES } \\
\hline & & & $0 \%$ & $10 \%$ & $20 \%$ & $30 \%$ & $40 \%$ & $50 \%$ & $0 \%$ & $10 \%$ & $20 \%$ & $30 \%$ & $40 \%$ & $50 \%$ \\
\hline \multirow{3}{*}{ Scenario 1} & $e_{s}^{\text {inst }}$ & [GWh] & 9.72 & 12.54 & 15.95 & 21.29 & 42.23 & 63.84 & 0.03 & 0.03 & 0.04 & 0.05 & 0.05 & 0.05 \\
\hline & $p_{i}^{\text {inst,inj }}, p_{o}^{\text {inst,off }}$ & {$[\mathrm{GW}]$} & 2.01 & 2.63 & 3.50 & 4.30 & 5.96 & 7.61 & 0.10 & 0.10 & 0.11 & 0.11 & 0.11 & 0.11 \\
\hline & E2P ratio & {$[\mathrm{h}]$} & 4.83 & 4.78 & 4.56 & 4.95 & 7.08 & 8.39 & 0.24 & 0.27 & 0.36 & 0.47 & 0.46 & 0.46 \\
\hline & $e_{s}^{\mathrm{Inst}}$ & {$[\mathrm{GWh}]$} & 8.70 & 8.70 & 8.70 & 8.70 & 8.70 & 8.70 & 0.03 & 0.12 & 0.35 & 0.44 & 0.51 & 0.49 \\
\hline \multirow[t]{2}{*}{ Scenario 2} & $p_{i}^{\text {inst, inj }}, p_{o}^{\text {inst }, \text { off }}$ & {$[\mathrm{GW}]$} & 1.94 & 2.26 & 2.64 & 2.42 & 2.54 & 3.12 & 0.11 & 0.20 & 0.45 & 0.55 & 0.62 & 0.62 \\
\hline & E2P ratio & {$[\mathrm{h}]$} & 4.49 & 3.85 & 3.29 & 3.60 & 3.43 & 2.8 & 0.25 & 0.58 & 0.78 & 0.81 & 0.82 & 0.79 \\
\hline \multirow{3}{*}{ Scenario 3} & $e_{s}^{\text {inst }}$ & {$[\mathrm{GWh}]$} & 8.70 & 8.70 & 8.70 & 8.70 & 8.70 & 8.70 & 0.86 & 1.58 & 1.61 & 4.34 & 7.05 & 10.29 \\
\hline & $p_{i}^{\text {inst,inj }}, p_{o}^{\text {inst,off }}$ & {$[\mathrm{GW}]$} & 1.35 & 1.62 & 2.13 & 1.66 & 1.55 & 1.71 & 0.78 & 1.06 & 1.19 & 2.26 & 3.47 & 4.56 \\
\hline & $\mathrm{E} 2 \mathrm{P}$ ratio & {$[\mathrm{h}]$} & 6.43 & 5.37 & 4.09 & 5.23 & 5.62 & 5.09 & 1.10 & 1.49 & 1.35 & 1.92 & 2.03 & 2.26 \\
\hline
\end{tabular}

\subsection{Reserve provision}

Fig. 6 shows the average reserve provision by the different generation and storage technologies for the different scenarios.

In the reference scenario, upward FCR is provided by online conventional generators that have head room available to provide this reserve. At low RES targets it is mainly provided by mid load plants, while at high RES targets a significant share is provided by high peak load plants. The different storage scenarios show that when storage is available, BES is about the sole provider, with PHS providing a minor share in scenario 2. In contrast to conventional generation technologies and PHS, BES does not have to be committed to provide upward FCR. In the reference scenario, and in the different storage scenarios at low RES targets, downward FCR is provided by online generation capacity, as they can provide this service fairly easy by ramping down. At high RES targets, and when storage is available to be installed, storage provides the largest share of downward FCR. As such, no conventional generators have to stay online to solely provide this service, especially taking into account the monthly contract periods. In scenario 1 and 2 both PHS and BES provide downward FCR, with the latter providing the major share, while at reduced energy-related BES investment costs it takes over PHS's share.

In the reference scenario upward aFRR is provided by online high peak load plants, while storage is the major upward aFRR provider in the different storage scenarios. In scenario 1 PHS is the main provider, while in scenario 2 its share decreases at the expense of BES, and upward aFRR provision is shared. In scenario $3 \mathrm{BES}$ is the main provider. Similar to FCR, at low RES targets the downward component is provided by ramping down base and mid load plants that are online most of the time anyway. When storage is available, and at higher RES targets, base and mid load power plants would not constantly be online anymore following efficient scheduling. Here, PHS provides the largest share in scenario 1, while both PHS and BES provide a large share in scenario 2 and 3, with BES becoming downward aFRR's main provider at high RES targets.

If storage resources are not available to be installed, upward mFRR is provided by high peak load generators, as they do not have to be committed at part-load but can start up in time. In storage scenario 1, the share of PHS of this energy-intensive reserve increases with the RES target. When the PHS energy capacity is limited, peak and 
high peak load plants again provide the largest share with PHS providing the remaining upward mFRR, and at lower BES energy-related investment cost they may provide a share of upward mFRR as well at the expense of high peak load generators. Again, downward mFRR is mostly provided by online conventional generators. When storage resources are available they provide an increasing share of downward mFRR as the RES target increases, since the amount of conventional generators that have to remain online for a month can be decreased. At current costs PHS is about the sole storage technology providing downward $\mathrm{mFRR}$, while in scenario 3 BES provides a significant share as well.

\section{Conclusions}

In the context of RES-driven power systems, short-term operating constraints and requirements are important to consider during long-term planning analyses. They are key drivers for flexibility, but large problem sizes and computation times have limited the extent to which they are included in policy and planning models. We present the development of a combined, and simultaneously solved, long-term investment and short-term operation model with high temporal and operational detail. It decides on the installed generation and storage capacities to cover the demand for energy and reserve capacity at lowest cost, given short-term operational constraints and residual demand variations, for increasing RES generation targets. Short-term operation is modeled through a continuous relaxation of the technology-clustered formulation of the UC problem. This allows for a better insight in the role and value of storage as flexibility source.

The availability of storage resources lowers total system cost. We show that this can be explained through its contribution to compenstate the system's expected and unexpected variability, and because less RES capacity needs to be installed to reach the imposed RES targets. First, storage has the ability to compensate the former by storing base load and RES generation in times of low residual demand, and by partly replacing peak and high peak generation in times of high residual demand. Second, storage has the ability to compensate the latter by providing reserves, thereby reducing the need for inefficient scheduling to accommodate must-run (partly-loaded) conventional generators to provide spinning reserve. Third, less RES needs to be installed to reach RES targets, as excess RES generation that otherwise had to be curtailed can now be stored, or simply generated to be consumed since the incompressible part of supply is lower.

The detailed modeling of frequency control allows for two main conclusions. First, independent from the storage scenario BES provides a significant share of FCR, while it is only when PHS is geographically constrained that BES provides significant shares of aFRR as well. Finally, when in addition the cost for the energy storage subsystem decreases, BES appears to be well-suited to provide FCR and aFRR, as well as mFRR. This provides conditions for which BES is a favorable technology to provide the different reserve products. Second, we quantitatively show how the interaction between energy and reserve markets leads to storage contributing to the provision of upward reserve at all RES targets, and to the provision of downward reserve only at high RES targets.

Results show that there is a need for both short-to-mid-term BES and mid-to-longterm PHS. PHS plants mainly provide energy services to the system, i.e., shifting energy in time, and energy-intensive reserve products, while BES systems mainly provide powerrelated reserve products. Even when the available PHS energy capacity would not be restricted by geographical conditions, BES is developed, and even when the energy-related 

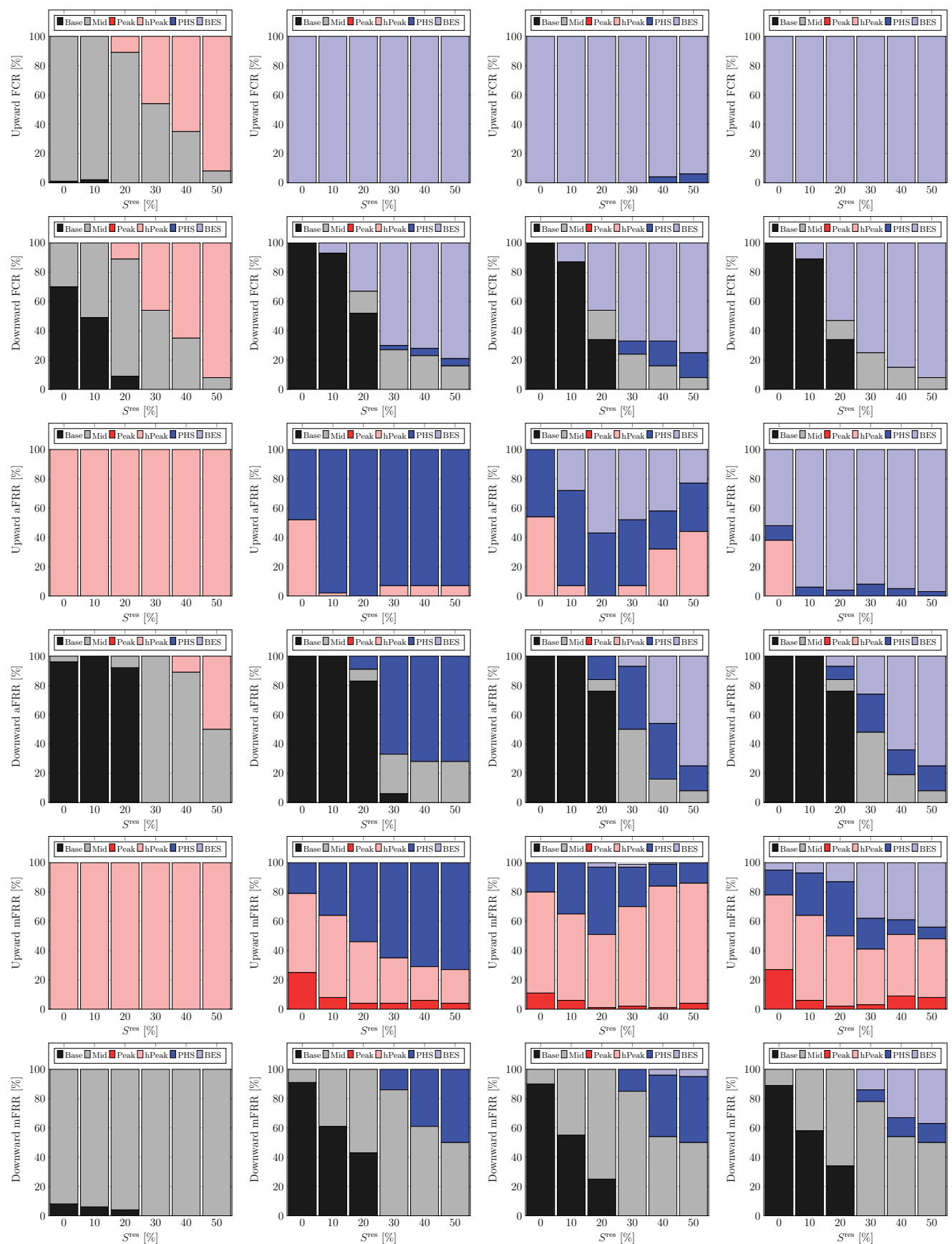

(a) Reference scenario.

(b) Storage scenario 1.

(c) Storage scenario 2.

(d) Storage scenario 3 .

Figure 6: Average reserve provision in different storage scenarios given a variety of RES targets. 
BES investment cost would decrease towards the future, the available PHS capacity is still fully developed. Although these sources compete to provide some flexibility services, they complement each other to meet the system's total demand for flexibility.

Furthermore, we conclude that a relationship is present between the imposed RES 630 target and installed flexible resources, independent from the analyzed scenario. This confirms that flexibility sources are to some extent interchangeable. It can be hypothesized that if due to capacity legacy or market design conventional flexible capacity remains operational in the system, this affects the development of alternative flexibility sources.

There are at least four topics for future work. First, an expansion of the geographical scope would allow to analyze the role of storage under different scenarios for the degree to which the considered system is interconnected. Second, an expansion of the flexibility sources would allow to study the impact of the price-responsiveness of the demand under different scenarios. Third, whereas the reservation of generation and storage capacity to provide frequency control is modeled, including the activation of reserves would present a valuable addition to the current state-of-the-art. Fourth, the developed model can be applied to inform a specific system's planners and policy-makers, and market players on the operation and investment in storage capacity. This would require including the current situation for the considered system through capacity legacy.

\section{Acknowledgment}

The authors would like to thank Benjamin F. Hobbs to co-host Tom Brijs at The Johns Hopkins University, and Research Foundation Flanders (FWO) for providing a travel grant. The authors would also like to thank Cedric De Jonghe and Frederik Geth for valuable comments, and the Department of Energy, Transportation, and Environment at DIW Berlin for hosting DIW Research Fellow Sauleh Siddiqui and Tom Brijs for discussing this research in October 2015.

\section{References}

[1] T. Brijs, D. Huppmann, S. Siddiqui, R. Belmans, Auction-based allocation of shared electricity storage resources through physical storage rights, J. of Energy Storage 7 (2016) 82-92. doi:10. 1016/j.est.2016.05.009.

[2] M. Gimeno-Guriérrez, Lacal-Arátegui, Assessment of the European potential for pumped hydropower energy storage based on two existing reservoirs, Renew. Energy 75 (2015) 856-868. doi: $10.1016 / j$.renene.2014.10.068.

[3] F. Geth, T. Brijs, J. Kathan, J. Driesen, R. Belmans, An overview of large-scale stationary electricity storage plants in Europe: Current status and new developments, Renew. \& Sustainable Energy Rev. 52 (2015) 1212-1227. doi:10.1016/j.rser.2015.07.145.

[4] C. Brivio, S. Mandelli, M. Merlo, Battery energy storage system for primary control reserve and energy arbitrage, Sustainable Energy, Grids and Networks 6 (2016) 152-165. doi:10.1016/j . segan. 2016.03 .004$.

[5] T. Brijs, F. Geth, S. Siddiqui, B. F. Hobbs, R. Belmans, Price-based unit commitment electricity storage arbitrage with piecewise linear price-effects, J. of Energy Storage 7 (2016) 52-62. doi: $10.1016 / j$.est. 2016.05 .005

[6] A. van Stiphout, K. De Vos, G. Deconinck, The impact of operating reserves on investment planning of renewable power systems, IEEE Trans. Power Syst. 32 (1) (2016) 378-388. doi:10.1109/TPWRS. 2016.2565058.

670 [7] M. Ventosa, A. Baíllo, A. Ramos, M. Rivier, Electricity market modeling trends, Energy Policy 33 (7) (2005) 897-913. doi:10.1016/j.enpol.2003.10.013. 
[8] A. van Stiphout, Short-term operational flexibility in long-term generation expansion planning, Ph.D. thesis, University of Leuven (KU Leuven) (2017).

[9] K. Poncelet, H. Hoschle, E. Delarue, A. Virag, W. D'haeseleer, Selecting representative days for capturing the implications of integrating intermittent renewables in generation expansion planning problems, IEEE Trans. Power Syst. PP (99) (2016) 1. doi:10.1109/TPWRS.2016.2596803.

[10] K. Poncelet, E. Delarue, D. Six, J. Duerinck, W. D'haeseleer, Impact of the level of temporal and operational detail in energy-system planning models, Appl. Energy 162 (2016) 631-643. doi: 10.1016/j.apenergy.2015.10.100.

11] S. Ludig, M. Haller, E. Schmid, N. Bauer, Fluctuating renewables in a long-term climate change mitigation strategy, Energy 36 (11) (2011) 6674-6685. doi:10.1016/j.energy.2011.08.021.

[12] B. S. Palmintier, M. D. Webster, Impact of operational flexibility on electricity generation planning with renewable and carbon targets, IEEE Trans. Sustainable Energy 7 (2) (2016) 672-684. doi: 10.1109/TSTE.2015.2498640.

[13] B. S. Palmintier, M. D. Webster, Impact of unit commitment constraints on generation expansion planning with renewables, in: IEEE PES General Meeting, 2011, pp. 1-7. doi:10.1109/PES.2011. 6038963.

[14] S. Pfenninger, A. Hawkes, J. Keirstead, Energy systems modeling for twenty-first century energy challenges, Renew. \& Sustainable Energy Rev. 33 (2014) 74-86. doi:10.1016/j.rser.2014.02.003.

690 [15] A. Shortt, J. Kiviluoma, M. O'Malley, Accommodating variability in generation planning, IEEE Trans. Power Syst. 28 (1) (2013) 158-169. doi:10.1109/TPWRS.2012.2202925.

[16] A. Pina, C. A. Silva, P. Ferrão, High-resolution modeling framework for planning electricity systems with high penetration of renewables, Appl. Energy 112 (2013) 215-223. doi:10.1016/j.apenergy . 2013.05.074.

[17] M. Black, G. Strbac, Value of storage in providing balancing services for electricity generation systems with high wind penetration, J. Power Sources 162 (2) (2006) 949-953. doi:10.1016/j. jpowsour.2005.07.020

[18] H. Lund, G. Salgi, The role of compressed air energy storage (CAES) in future sustainable energy systems, Energy Convers. and Management 50 (5) (2009) 1172-1179. doi:10.1016/j.enconman. 2009.01.032.

[19] A. Tuohy, M. O'Malley, Pumped storage in systems with very high wind penetration, Energy Policy 39 (4) (2011) 1965-1974. doi:10.1016/j.enpol.2011.01.026.

[20] P. Denholm, M. Hand, Grid flexibility and storage required to achieve very high penetration of variable renewable electricity, Energy Policy 39 (3) (2011) 1817-1830. doi:10.1016/j.enpol. 2011. 01.019.

[21] N. Zhang, C. Kang, D. S. Kirschen, Q. Xia, W. Xi, J. Huang, Q. Zhang, Planning pumped storage capacity for wind power integration, IEEE Trans. Sustainable Energy 4 (2) (2013) 393-401. doi: 10.1109/TSTE.2012.2226067.

[22] C. O'Dwyer, D. Flynn, Using energy storage to manage high net load variability at sub-hourly time-scales, IEEE Trans. Power Syst. 30 (4) (2015) 2139-2148. doi:10.1109/TPWRS. 2014.2356232

[23] W.-P. Schill, Residual load, renewable surplus generation and storage requirements in Germany, Energy Policy 73 (2014) 65-79. doi:10.1016/j.enpol.2014.05.032.

[24] S. Weitemeyer, D. Kleinhans, T. Vogt, C. Agert, Integration of renewable energy sources in future power systems: The role of storage, Renew. Energy 75 (2015) 14-20. doi:10.1016/j.renene.2014. 09.028.

[25] H. Bludszuweit, J. A. Domínguez-Navarro, A probabilistic method for energy storage sizing based on wind power forecast uncertainty, IEEE Trans. Power Syst. 26 (3) (2011) 1651-1658. doi 10.1109/TPWRS. 2010.2089541.

[26] Y. V. Makarov, P. Du, M. C. W. Kintner-Meyer, C. Jin, H. F. Illian, Sizing energy storage to accommodate high penetration of variable energy resources, IEEE Trans. Sustainable Energy 3 (2012) 34-40. doi:10.1109/TSTE.2011.2164101.

[27] H.-I. Su, A. El Gamal, Modeling and analysis of the role of energy storage for renewable integration: Power balancing, IEEE Trans. Power Syst. 28 (4) (2013) 4109-4117. doi:10.1109/TPWRS.2013. 2266667.

[28] B. Hartmann, A. Dán, Methodologies for storage size determination for the integration of wind power, IEEE Trans. Sustainable Energy 5 (1) (2014) 182-189. doi:10.1109/TSTE. 2013.2278423.

[29] C. Bussar, P. Stöcker, Z. Cai, L. Moraes Jr., D. Magnor, P. Wiemes, N. van Bracht, A. Moser, D. U. Sauer, Large-scale integration of renewable energies and impact on storage demand in a European renewable power system of 2050 Sensitivity study, J. of Energy Storage 6 (2016) 1-10. doi: $10.1016 /$ j.est.2016.02.004. 
[30] M. Haller, S. Ludig, N. Bauer, Bridging the scales: A conceptual model for coordinated expansion of renewable power generation, transmission and storage, Renew. \& Sustainable Energy Rev. 16 (5) (2012) 2687-2695. doi:10.1016/j.rser.2012.01.080.

[31] P. Yang, A. Nehorai, Joint optimization of hybrid energy storage and generation capacity with renewable energy, IEEE Trans. Smart Grid 5 (4) (2014) 1566-1574. doi:10.1109/TSG.2014.2313724.

[32] A. Zerrahn, W.-P. Schill, A greenfield model to evaluate long-run power storage requirements for high shares of renewables (2015).

URL https://www.diw.de/documents/publikationen/73/diw_01.c.498475.de/dp1457.pdf

[33] D. J. Swider, Compressed air energy storage in an electricity system with significant wind power generation, IEEE Trans. Energy Convers. 22 (1) (2007) 95-102. doi:10.1109/TEC.2006.889547.

[34] E. Hale, B. Stoll, T. Mai, Capturing the impact of storage and other flexible technologies on electric system planning, Tech. Rep. NREL/TP-6A20-65726, National Renewable Energy Laboratory (NREL), Golden, Colorado, USA (2016). URL http://www.nrel.gov/docs/fy16osti/65726.pdf

35] G. Strbac, M. Aunedi, D. Pudjianto, P. Djapic, F. Teng, A. Sturt, D. Jackravut, R. Sansom, V. Yufit, N. Brandon, Strategic assessment of the role and value of energy storage systems in the UK low carbon energy future, Tech. rep., Energy Futures Lab, Imperial College London, London, UK (2012).

URL https://www.carbontrust.com/media/129310/energy-storage-systems-role-value-strategic-assessment. pdf

[36] D. Pudjianto, M. Aunedi, P. Djapic, G. Strbac, Whole-systems assessment of the value of energy storage in low-carbon electricity systems, IEEE Trans. Smart Grid 5 (2) (2014) 1098-1109. doi: 10.1109/TSG.2013.2282039.

[37] F. J. de Sisternes, J. D. Jenkins, A. Botterud, The value of energy storage in decarbonizing the electricity sector, Appl. Energy 175 (2016) 368-379. doi:10.1016/j.apenergy.2016.05.014.

[38] K. Van Den Bergh, K. Bruninx, E. Delarue, W. D'haeseleer, LUSYM: A unit commitment model formulated as a mixed-integer linear program (2016).

URL_https://www.mech.kuleuven.be/en/tme/research/energy_environment/Pdf/ wpen2014-07-2.pdf

760 [39] B. S. Palmintier, M. D. Webster, Heterogeneous unit clustering for efficient operational flexibility modeling, IEEE Trans. Power Syst. 29 (3) (2014) 1089-1098. doi:10.1109/TPWRS.2013.2293127.

[40] P. J. Ramirez, D. Papadaskalopoulos, G. Strbac, Co-optimization of generation expansion planning and electric vehicles flexibility, IEEE Trans. Smart Grid 7 (3) (2016) 1609-1619. doi:10.1109/TSG. 2015.2506003

765 [41] B. Palmintier, Flexibility in generation planning: Identifying key operating constraints, in: Power Systems Computation Conference (PSCC), Wroclaw, Poland, 2014. doi:10.1109/PSCC.2014. 7038323.

[42] J. Tant, F. Geth, D. Six, P. Tant, J. Driesen, Multiobjective battery storage to improve PV integration in residential distribution grids, IEEE Trans. Sustainable Energy 4 (1) (2013) 182-191. doi:10.1109/TSTE. 2012.2211387.

[43] Elia, Evolution of ancillary services needs to balance the Belgian control area towards 2018, Tech. rep., Brussels (2013).

URL http://www.elia.be/ /media/files/Elia/Grid-data/Balancing/Reserves-Study-2018.pdf

[44] ENTSO-E, Supporting Document for the Network Code on Load-Frequency Control and Reserves, Tech. rep., Brussels, Belgium (2013).

URL https://www.entsoe.eu/fileadmin/user_upload/_library/resources/LCFR/130628-NC_ LFCR-Supporting_Document-Issue1.pdf

[45] Elia, Studie over de nood aan 'adequacy' en aan 'flexibiliteit' in het Belgische elektriciteitssyteem, Tech. rep., Brussels, Belgium (2016). URL http://www.elia.be/ AdequacyReport_2017-2027_NL.pdf

[46] S. Simoes, W. Nijs, P. Ruiz, A. Sgobbi, D. Radu, P. Bolat, C. Thiel, S. Peteves, The JRC-EUTIMES model: Assessing the long-term role of the SET plan energy technologies, Tech. rep., JRC Scientific and Policy Reports, Petten, The Netherlands (2013). doi:10.2790/97596. URL https://ec.europa.eu/jrc/en/publication/eur-scientific-and-technical-research-reports/ jrc-eu-times-model-assessing-long-term-role-set-plan-energy-technologies

[47] A. Schröder, F. Kunz, J. Meiss, R. Mendelevitch, C. von Hirschhausen, Data documentation: Current and prospective costs of electricity generation until 2050, Tech. rep., DIW Berlin, Berlin, Germany (2013) 
URL https://www.diw.de/documents/publikationen/73/diw_01.c.424566.de/diw_datadoc_ 2013-068.pdf

[48] C. De Jonghe, E. Delarue, R. Belmans, W. D'haeseleer, Determining optimal electricity technology mix with high level of wind power penetration, Appl. Energy 88 (6) (2011) 2231-2238. doi: $10.1016 / j$.apenergy.2010.12.046.

49] 50Hertz, Amprion, Elia, Tennet, TransnetBW, Potential cross-border balancing cooperation between the Belgian, Dutch and German electricity transmission system operators, Tech. rep., EBridge Consulting GmbH, Bonn, Germany (2014).

URL http://www.elia.be/ /media/files/Elia/users-group/141008_Final_report.pdf

[50] Elia, Data download page (2016).

URL http://www.elia.be/en/grid-data/data-download

800 [51] ENTSO-E, Consumption data (2016)

URL https://www.entsoe.eu/data/data-portal/consumption/Pages/default.aspx 